\title{
Validity of the dispersion relations in magnetotellurics: Part I - theory
}

\author{
Nikita Zorin ${ }^{1,2}$, Elena Aleksanova ${ }^{1}$, Hisayoshi Shimizu ${ }^{2^{*}}$ (1) and Denis Yakovlev ${ }^{1}$
}

\begin{abstract}
Application of the dispersion relations (DR) in magnetotellurics (MT) is an efficient tool of post-processing and quality assessment of broadband field data. The main limitation of the approach is that it requires the observed transfer functions to be causal and minimum-phase (MP), which is formally secured only for 1-D and some types of 2-D impedances. As a consequence, many MT practitioners involuntarily restrict the DR application to apparent resistivity curves acquired in relatively simple geological conditions. In the present research, we show how an inherently non-MP or non-causal transfer function could be recognized, and propose a universal technique, which makes it possible to correctly apply the DR virtually to any set of field MT data.
\end{abstract}

Keywords: Magnetotellurics, Dispersion relations, Causality, Minimum phase, Phase out of quadrant

\section{Application of the DR in magnetotellurics Introduction}

Magnetotellurics (MT) is a passive geophysical method used to infer the Earth's resistivity structure from the measurements of the natural electric (telluric) and magnetic field components either at Earth's surface or at the seafloor (Simpson and Bahr 2005). This task is inherently unstable with respect to small errors in the initial data, hence its immediate solution may yield results far from reality. As a consequence, in order to get any reliable and meaningful results out of MT measurements it is essential to use a priori information (Berdichevsky and Dmitriev 2002).

Application of a priori knowledge to constrain the inverse problem is not limited to reliance on known geological, geophysical or other background information when inverting MT data. In fact, it begins already at the stage of the time-series processing. Indeed, mere understanding of MT signals' usual behavior helps an experienced geophysicist to find the least noisy part of the record; prior knowledge of real data distribution models

\footnotetext{
${ }^{*}$ Correspondence: shimizu@eri.u-tokyo.ac.jp

2 Earthquake Research Institute, The University of Tokyo, 1-1-1, Yayoi,

Bunkyo-ku, Tokyo 113-0032, Japan

Full list of author information is available at the end of the article
}

allows implementation of advanced algorithms of data processing (Chave 2017); the smoothness property of MT curves validates using smoothing splines for their robust estimation (Berdichevsky and Dmitriev 2002); and so on. In this paper, we discuss the applicability of the dispersion relations (DR) in magnetotellurics-a powerful but highly controversial a priori assumption, widely used for detection and elimination of noisy/biased MT data (Berdichevsky and Dmitriev 2008). Our ultimate goal is to make the DR technique practically applicable to any given broadband magnetotelluric measurement.

\section{Causal and minimum-phase functions}

To begin with, we note that all available analytic conclusions concerning the DR validity in magnetotellurics (viz., Weidelt 1972, 2003; Weidelt and Kaikkonen 1994; Berdichevsky and Pokhotelov 1997) are derived using the methods of the theory of functions of a complex variable. The main idea of this approach is to regard the frequency as a complex variable $\Omega=\omega+i z$ and consider the properties of transfer functions not only at real frequencies $\omega$, but in the whole $\Omega$-plane. For clarity, here we restrict ourselves by listing the main properties of physically realizable response functions to be used in the further analysis. For more details on the subject, the reader is referred to the classic treatises of Bode (1945), Chapters 2, 7, 
13-14); Titchmarsh (1948), Chapter 5); Landau and Lifshitz (1958), section 122; Landau and Lifshitz 1960, section 62); Nussenzweig (1972), Chapter 1) and others. A brief review of the problem with regard to electromagnetic prospecting methods could also be found in Zorin and Alekseev (2018).

Let $\quad F(\omega)=|F(\omega)| e^{i \arg [F(\omega)]}=\operatorname{Re}[F(\omega)]+i \operatorname{Im}[F(\omega)]$ be the Fourier spectrum of a real-valued signal $f(t)$. The first property of $F(\Omega)$ is that it is well-behaved (analytic) in the whole $\Omega$-plane except of some singular points, where it takes on infinite values. Such points are called "poles" and their exclusive nature lies in the fact that they almost entirely (to within a constant) determine the behavior of $F(\Omega)$ at all other frequencies. Another important points called "zeros" are defined as those where $F(\Omega)=0$.

The second property of $F(\Omega)$ to be noted is the reflection symmetry, i.e.,

$$
F\left(-\Omega^{*}\right)=F^{*}(\Omega) .
$$

This equation says that $F(\Omega)$ takes complex conjugate values at two points of the $\Omega$-plane which are mirror images of each other across the imaginary axis. In particular, since the complex conjugate of an infinite number is also infinite, and complex conjugate of 0 is also 0 , all the poles and zeros of $F(\Omega)$ located at either right or left half $(\omega \neq 0)$ of the complex plane turn out to be reflected to the other half (Fig. 1a).

Now assume that $f(t)$ is not just an arbitrary signal, but a physically realizable response function observed at the output of a linear, passive, time-invariant system ("black box"), which is excited by the Dirac delta impulse $\delta(t)$. According to the principle of causality (an effect cannot occur before its cause), $f(t)=0$ for any $t<0$. For the harmonic time factor $e^{i \omega t}$ used in the paper, this statement is equal to the requirement that all the poles of
$F(\Omega)$ are restricted to be either in the upper half-plane $(z>0)$ or at the real frequency axis $\omega(z=0)$. As a consequence, the causality concept could be translated into the frequency domain using the following definition: the transfer function $F(\omega)$ is called "causal" if $F(\Omega)$ has no poles in the lower half-plane (Fig. 1b).

Another concept widely employed in the theory of the dispersion relations is that of the minimum phase. The function $F(\omega)$ is called "minimum-phase" (MP) if $F(\Omega)$ has neither poles nor zeros in the lower halfplane (Fig. 1c). From this definition follows the two major properties of MP functions. The first one lies in the fact that every MP function is causal (while not every causal function is MP), and the second one indicates that if $F(\omega)$ is MP, then its complex logarithm $\ln F(\omega)=\ln |F(\omega)|+i \arg [F(\omega)]$ has no poles in the lower half-plane and, hence, is also a causal function.

\section{Dispersion relations of the two kinds}

Depending on the given field of study and the particular mathematical representation form, an integral relation between the components of a complex function may be referred to in the literature as the dispersion relation, Kramers-Kronig relation, Bode relation, Sokhotski-Plemelj formula and the Hilbert transform (Zorin and Alekseev 2018). Following Berdichevsky and Dmitriev (2008), in this paper, we make the distinction between the dispersion relation of the first kind (DR-I), which connects the real and imaginary parts of a causal function:

$$
\operatorname{Im}[F(\omega)]=\frac{\pi}{2} \cdot \frac{d \operatorname{Re}[F]}{d \ln \omega} * B(\ln \omega)=\operatorname{DR}[\operatorname{Re}[F(\omega)]]
$$

and the dispersion relation of the second kind (DR-II), which connects the phase and logarithmical amplitude of a minimum-phase (MP) function:

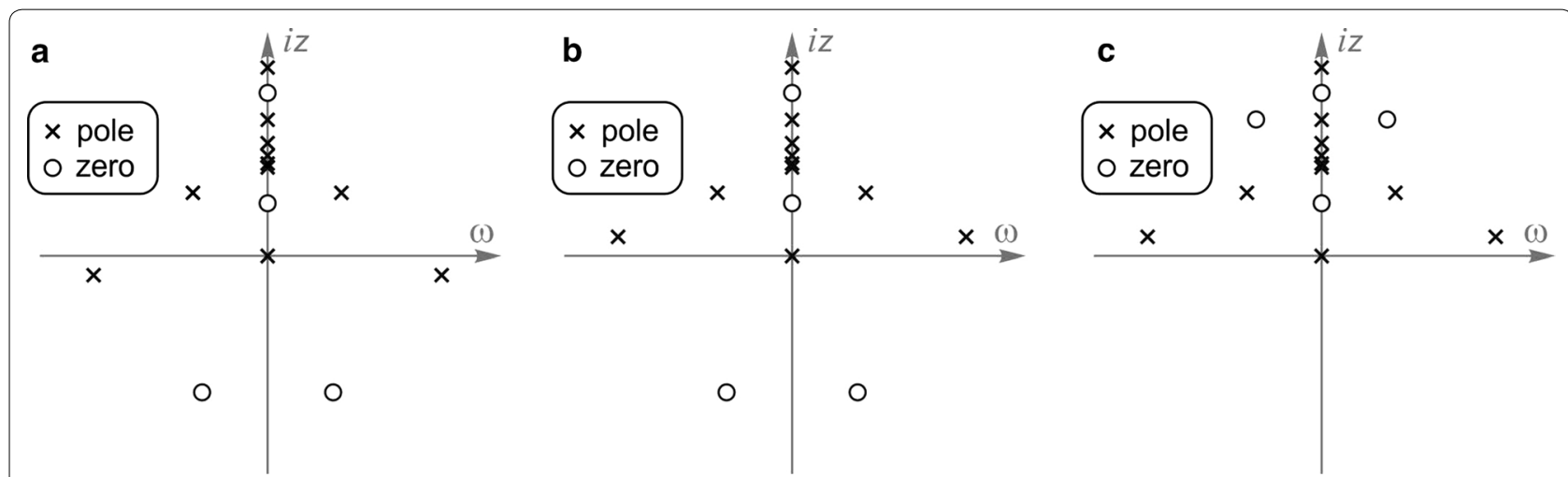

Fig. 1 Possible locations of poles and zeros in the complex frequency plane for: a spectrum of a real-valued signal; b causal transfer function; c MP transfer function 


$$
\arg [F(\omega)]=\frac{\pi}{2} \cdot \frac{d \ln |F|}{d \ln \omega} * B(\ln \omega)=\mathrm{DR}[\ln |F(\omega)|],
$$

where the asterisk denotes the convolution operation, and the Bode kernel

$$
B(u)=\frac{2}{\pi^{2}} \ln \operatorname{coth} \frac{|u|}{2}
$$

is normalized in such a way that its integral over infinite limits is equal to one (Bode 1945).

Note that since one can always switch polarity of a measured signal without changing its form and amplitude, the DR-II is generally correct only up to within an additive constant $\pi$. It is also essential to bear in mind that for the proper use of the dispersion relations in the MT method one should carefully choose their target functions. Though this issue is described in more detail further, it would be appropriate to underline here a trivial but important fact that any DR is originally supposed to be valid within one given complex function. For instance, provided that the causality and MP conditions are satisfied, Eq. (2) may immediately be applied to the components of the impedance tensor $\hat{Z}$, admittance tensor $\hat{Y}=1 / \hat{Z}$ and Wiese-Parkinson (tipper) matrix $\hat{W}$, magnetic tensor $\hat{M}$ and telluric tensor $\hat{T}$, frequency-normalized tensors $\hat{C}=\hat{Z} / i \omega \mu_{0}$ and $\hat{Z}^{n}=\hat{Z} / \sqrt{i \omega \mu_{0}}$, etc. The same naturally holds for the complex apparent resistivity functions $\rho_{x y}=Z_{x y}^{2} / i \omega \mu_{0}$ and $\rho_{y x}=-Z_{y x}^{2} / i \omega \mu_{0}$. However, the traditional MT practice is based on consideration of the apparent resistivity amplitude along with the impedance phase, i.e., the spectral components of two different functions. Immediate application of the DR-II to such data requires Eq. (2b) to be modified as follows:

$$
\begin{aligned}
& \arg \left[Z_{x y}(\omega)\right]=\frac{\pi}{4} \cdot \frac{d \ln \left|\rho_{x y}\right|}{d \ln \omega} * B(\ln \omega)+\frac{\pi}{4}, \\
& \arg \left[Z_{y x}(\omega)\right]=\frac{\pi}{4} \cdot \frac{d \ln \left|\rho_{y x}\right|}{d \ln \omega} * B(\ln \omega)-\frac{3 \pi}{4} .
\end{aligned}
$$

Another practical issue arises if one decides to apply the DR-I (Eq. 2a) to the impedance tensor components without their frequency normalization. As opposed to the dispersion relation of the second kind, the DR-I application implies linear vertical scaling of the considered functions, which is substantially undescriptive for the components of $\hat{Z}$. The main reason is that $|\operatorname{det} \hat{Z}(\omega)|$ is a rapidly ( $\sim \omega$ over a half-space) increasing function on the whole domain of its definition, which effectively conceals the influence of the underlying geoelectric medium on the $\hat{Z}$ components reviewed on a lin-log plot. On the other hand, tensor $\hat{C}=\hat{Z} / i \omega \mu_{0}$ turns out to be "overnormalized" in this sense-its determinant amplitude decreases as fast as $\omega$ over a half-space, and its components appear to be equally useless for the DR-I application as those of the impedance tensor. A natural way out is to employ Basokur's normalization $\hat{Z}^{n}=\hat{Z} / \sqrt{i \omega \mu_{0}}$, which ensures that any observed frequency dependence is only due to geoelectric structures and makes the presented data informative (Basokur 1999). Indeed, as seen from Fig. 2, the real and imaginary parts representation of $Z_{x y}^{n}$ is much more descriptive than that of $Z_{x y}$ or $C_{x y}$, and qualitatively resembles the conventional ( $\rho_{x y}$ amplitude and $Z_{x y}$ phase) representation of the same MT data. Similarly, should the DR-I be applied to the admittance tensor $\hat{Y}$, it better be applied to $\hat{Y}^{n}=1 / \hat{Z}^{n}=\hat{Y} \sqrt{i \omega \mu_{0}}$ instead. Note that $\left|\rho_{i j}\right|=\left|Z_{i j}^{n}\right|^{2}$, hence all the components of $\hat{Z}^{n}$ has the units of $\sqrt{\Omega \cdot m}$, while the components of $\hat{Y}^{n}$ are measured in $\sqrt{S / m}$. Tensors $\hat{M}, \hat{T}, \hat{W}$, and all other relative (and thereby unitless) MT transfer functions do not require frequency normalization prior to the DR-I application to their components.

\section{Physical meaning of the dispersion relations}

The DR formulation could be manifold-for more details and examples as applied to MT problems the reader is referred to the papers of Weidelt (1972), Kunetz (1972), Boehl et al. (1977), Fischer and Schnegg (1980), Marcuello et al. (2005), and others. However, the ones given above have several useful advantages, among which we should highlight their simplicity both in formalism and practical application for smooth functions of $\ln \omega$, as well as the presence of an illustrative physical meaning. Indeed, since the Bode kernel (Eq. 3) represents an infinite symmetric averaging filter (Fig. 3), Eq. (2a) implies that the imaginary part of a causal function is equal to the smoothed derivative of its real part on a log-frequency plot times $\pi / 2$. Similarly, from Eq. (2b) follows that the phase of a MP function is proportional with the same coefficient to the smoothed derivative of its amplitude plotted on log-log scale.

From Fig. 3 it is also seen that the effective width of the Bode kernel is about one decade of frequency: 74\% of its weight lies within the region $[-\ln \sqrt{10} ; \ln \sqrt{10}]$ and $\sim 92 \%$-within the region $[-\ln 10 ; \ln 10]$. As soon as any geophysical measurement is essentially bounded by some finite frequency range, two major conclusions follow. On the one hand, the boundary values of imaginary part (phase) constrains the behavior of the corresponding real part (amplitude) up to roughly half of a decade outside the measured frequency band (Fig. 4), which clearly substantiates the advantage of the combined amplitude + phase (or real+imaginary parts) data inversion methods. On the other hand, within the inner section of 

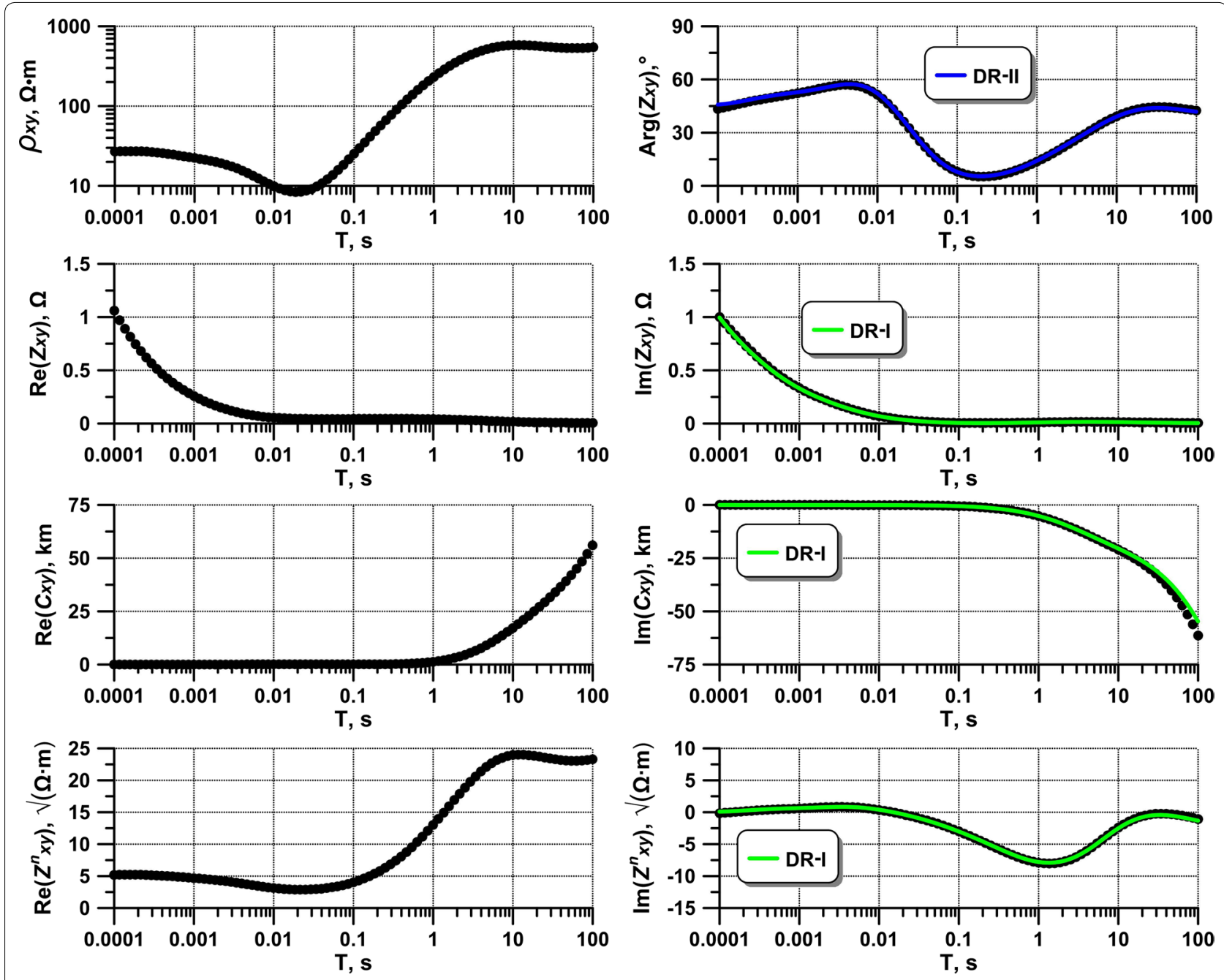

Fig. 2 The DR application to a given transfer function $Z_{x y}$ expressed in the form of (from top to bottom): apparent resistivity $\rho_{x y}$ and impedance phase $\arg \left[Z_{x y}\right]$; real and imaginary parts of $Z_{x y}$; real and imaginary parts of $C_{x y}=Z_{x y} / i \omega \mu_{0}$; real and imaginary parts of $Z_{x y}^{n}=Z_{x y} / \sqrt{i \omega \mu_{0}}$

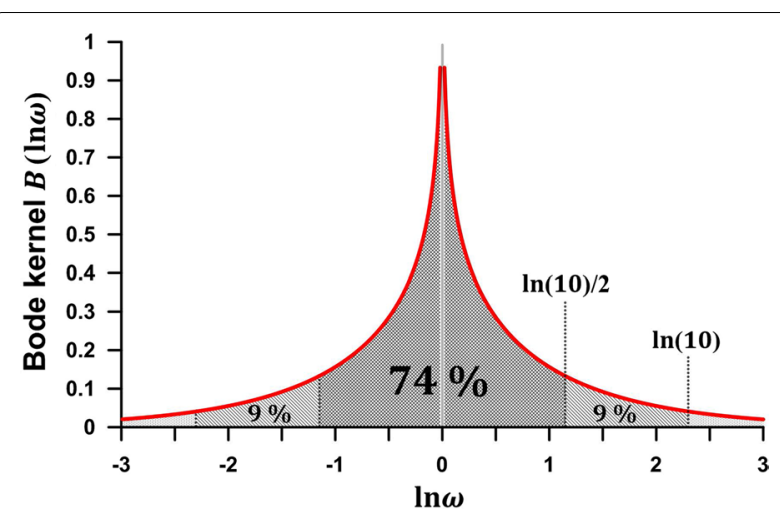

Fig. 3 Bode kernel function. The areas of the highlighted sections are given as percentage of the total area under the red curve the frequency band the imaginary part (phase) contains no unique information and could be fully recovered from the real part (amplitude) of the same function, which may be effectively used to improve the quality of measured data or check their self consistency, as shown below.

\section{Improving the data quality}

The transfer function estimation routine in magnetotellurics could generally be divided into two steps. The first one consists of automatic processing of raw MT data (time series) with the help of one or another robust statistical algorithm and is described in detail elsewhere (see Chave 2012, 2017 for a review). This step generally requires the measured electric and/or magnetic time series at one or several MT sites, frequency 


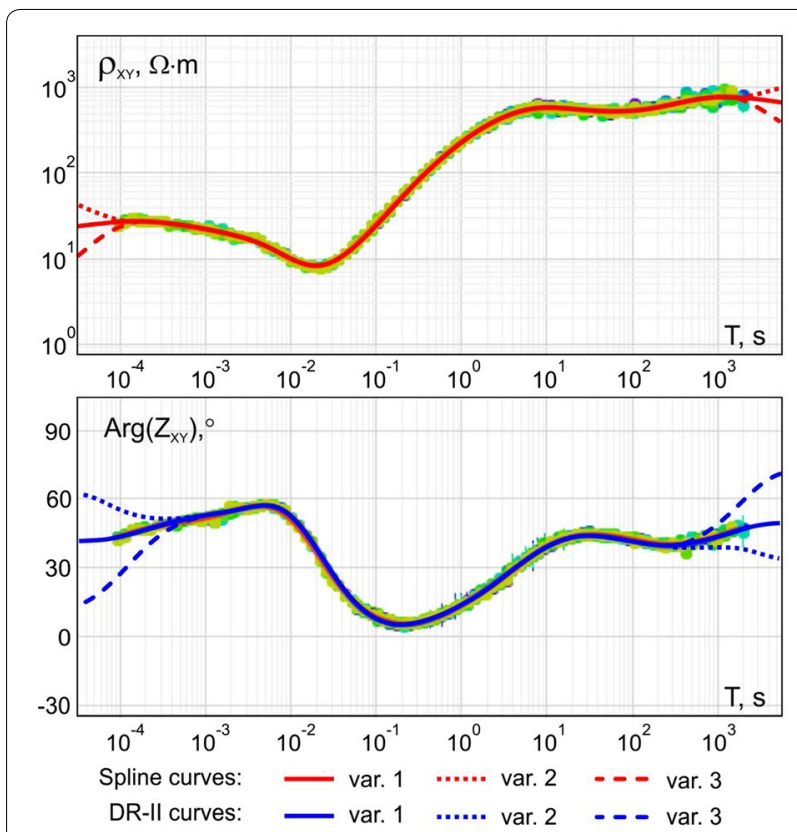

Fig. 4 An illustration of how the DR-II constrains the behavior of an MT transfer function outside the measured frequency band. The apparent resistivity extrapolations plotted with dashed and dotted lines are found to be inconsistent with the available phase data

characteristics (calibrations) of the equipment used, and some a priori assumptions regarding the distribution and noise contamination of raw data. At the output of the procedure one obtains a set of independent estimations (less often-a single estimation) of a given transfer function and the corresponding errors at each frequency. Most of the MT data used in the present research were processed with the help of either the classic commercial software package SSMT-2000 (Phoenix Geophysics Ltd., Canada) or the modern Epi-Kit software (Nord-West Ltd., Russia), which employs several advanced estimation procedures described in Epishkin (2016).

The second step, usually referred to as post-processing, consists of finding the best way to approximate the separate estimations obtained earlier with smooth MT curves and appears to be a perfect candidate for the DR application (Boehl et al. 1977; Fischer and Schnegg 1980). In some cases this interactive step requires intervention of an experienced specialist, but may significantly suppress the influence of noisy data and should be performed with all due care. Indeed, the perfect-quality data examples similar to that shown on Fig. 4 are rarely encountered in real practice-as a rule, many results of data processing turn out to be scattered and biased, even when using the robust algorithms with a remote reference site (Chave 2012).

An example of such data is given in Fig. 5, which considers a 4-day MT measurement C-02 (Additional file 1) taken in December, 2012 as part of the cooperative RussianUkrainian academic project at Baryatinskaya anomaly of crustal conductivity (Kulikov et al. 2018). The survey site is located in densely populated Western part of Russia, right

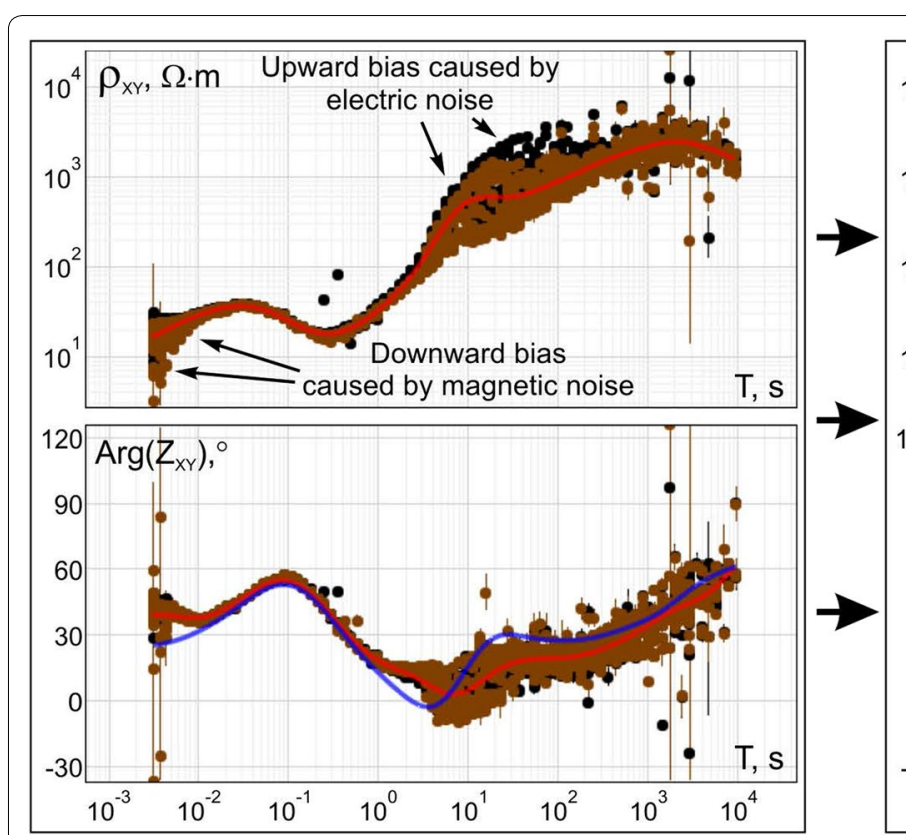

Raw data: $\phi \phi \phi$ "Remote H" processing

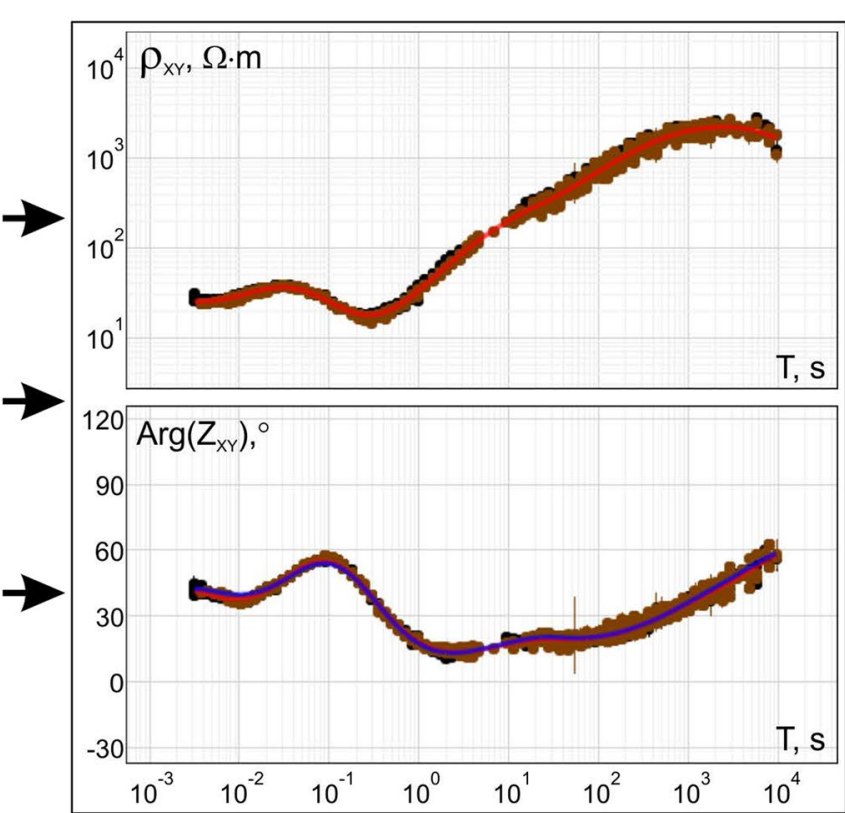

Curves: - spline - DR-II

Fig. 5 Post-processing of long-period MT data acquired in Western Russia 
between the DC-electrified sections of Moscow-Minsk and Moscow-Kiev railways, which is responsible for the notable upward bias at periods about 3-5 s and longer. In addition, at short periods the amplitude estimations are biased downwards due to increased sensor noise of the employed magnetic coils (MTC-50 by Phoenix Geophysics Ltd.) at the given frequencies. Application of the DR-II helps to find and remove the biased data estimations, thus greatly improving the resulting quality of the obtained impedance function (Fig. 5). In this procedure it could also be useful to consider several data processing methods, susceptible to different biases, together. Brown dots in Fig. 5 represent the results of the conventional "Remote $\mathrm{H}$ " data processing scheme, which effectively suppresses the bias caused by noise in electric channels (Chave 2012). The "noise-free" magnetic components for such processing were taken from the remote reference site at Aleksandrovka geophysical field camp (Aleksanova et al. 2018) located about $80 \mathrm{~km}$ away from the survey site. Black dots correspond to the "Local E" single-station processing scheme which assumes the local electric field to be noise-free and may effectively suppress the bias caused by noisy magnetic data (Sims et al. 1971). As seen from Fig. 5, both methods yield virtually identical results within the $10^{-2} \mathrm{~s}-10^{0} \mathrm{~s}$ period range, but provide different results for noisy data: "Local E" estimations are naturally more exposed to the industrial electric noise, while being less affected by the instrumental noise in the magnetic channels.

It is to be noted here that some authors (e.g., Larsen, 1989; Sutarno and Vozoff 1991; Ernst et al. 2001) propose making use of the smoothness and/or DR constraints already at the step of automatic data processing. Though such algorithms could definitely be effective in many practical cases, they also make rather difficult to control the actual reliability of the obtained results. For this reason, we are, so far, inclined to employ the MT processing codes yielding independent estimates for each given frequency, with the interactive application of the smoothing splines and dispersion relations during the post-processing routine (e.g., Fig. 5; see also another one detailed example of the procedure given in Appendix 1).

\section{Checking the data consistency}

The dispersion relations are found to be useful in many other MT applications, such as detection and adjustment of improper equipment calibrations, quality control of the numerical modeling, checking the consistency of MT transfer functions for quantitative interpretation, etc. One of the promising areas of the DR application in this regard is the issue of the source effect recognition (Shimizu et al. 2011), which could be briefly described as follows.

The fundamental assumption of the MT method is that in the non-conductive upper half-space there exists a quasi-uniform source giving rise to plane "waves" normally incident on the surface of the conductive Earth (Simpson and Bahr 2005). Notwithstanding that the actual sources of the magnetotelluric signal are in fact finite and manifold (Viljanen 2012), the plane-wave approximation remains mathematically correct as long as the skin depth for the induced currents is much less than the spatial scale of the external field variations (Berdichevsky and Dmitriev 2002; Utada 2018), which holds for most MT case studies. However, departures from this basic assumption are routinely encountered in practice, e.g.: in polar and equatorial regions owing to instabilities in the forms of the source fields (Simpson and Bahr 2005); in some resistive regions of the Earth crust due to short spatial scale source fields from Pc3-4 pulsations (Murphy and Egbert 2018); in any MT data measured at very long periods due to the influence of the diurnal Sq variations (Shimizu et al. 2011); etc. In all such cases the source effect (provided that it is strong enough to significantly affect the data) forces an MT response to "switch" at some frequency from being excited by the regular plane-wave source to an input signal of some other type, which naturally manifests itself by violation of the dispersion relations. Since the vertical magnetic field component is more sensitive to non-uniform source field contributions than horizontal MT components (Jones and Spratt 2002; Utada 2018), the tipper function $\hat{W}$ appears to be particularly vulnerable to the distorting influence of the source effect, as could be seen from two following examples.

Figure 6 shows the $W_{z x}$ component estimated by applying the EMTF code of Egbert and Booker (1986) to the 4-week MT measurement taken in September-October, 2012, at the station IAN-37 in Iowa, USA, as part of the EarthScope USArray project (Murphy and Egbert 2018). Figure 7 depicts the $W_{z y}$ component estimated by applying the BIRRP code of Chave and Thomson (2004) to the corrected for the principal Sq signatures 12-month data recorded in 2005-2006 at the geomagnetic observatory CBI in Chichijima, Japan (Shimizu et al. 2011). In both cases the DR-I application confirms the inconsistency of the data points (marked with red) within the period ranges considered by above authors as being heavily influenced by source effect. Such a result naturally prompts one to take some decisive action, e.g., exclude the inconsistent data estimations from the regular MT inversion (Marcuello et al. 2005) or implement a more realistic source model (Egbert and Booker 1989).

\section{Inverse dispersion relations}

The fundamental theory of physically realizable signals (Bode 1945; Titchmarsh 1948; Toll 1956) generally prescribes the existence of an equivalent pair of dispersion 


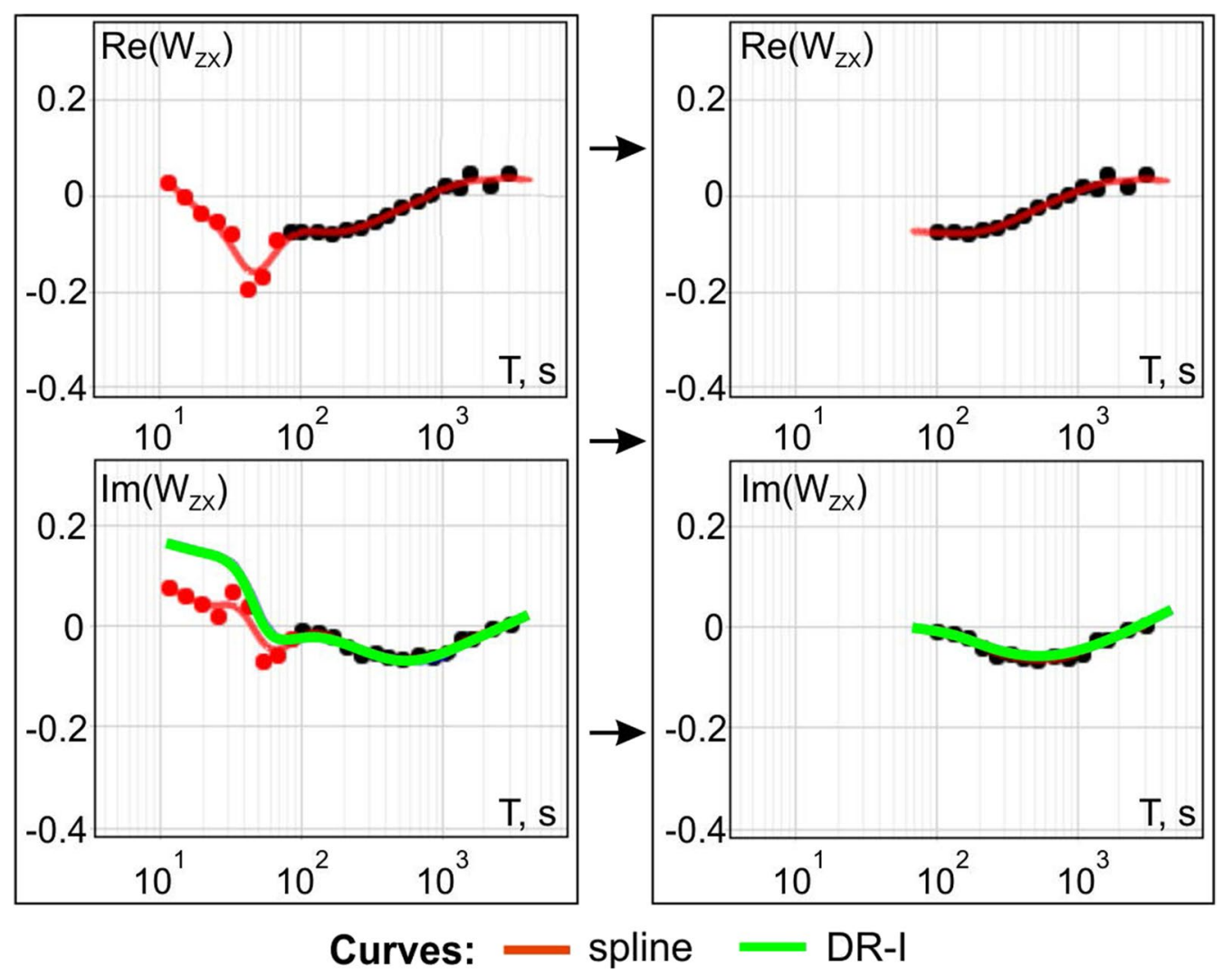

Fig. 6 Application of the DR-I to the tipper data acquired at site IAN-37 in lowa, USA; the data points distorted by Pc3-4 source effect are marked with red (modified after Murphy and Egbert 2018)

relations, which may be used not only to calculate the imaginary part (phase) from the real part (amplitude logarithm) of a causal (MP) spectrum, but also vice versa. The "inverse" dispersion relations employed for the latter calculation are less popular than the "forward" relations considered above since they define the real part (amplitude logarithm) of a transfer function only to within an arbitrary constant and are more difficult to implement. However, inasmuch as the impedance phase estimations are often less biased than those of the amplitude, application of the inverse DR-II could also be reasonable (Boehl et al. 1977; Sutarno and Vozoff 1991).

As in the case of the forward dispersion relations, the inverse DR formalism is manifold (see Bode 1945; Boehl et al. 1977; Fischer and Schnegg 1980; Zorin and Alekseev 2018). Here we give the following expressions, which apparently have the simplest form:

$$
\begin{aligned}
& \operatorname{Re}[F(\omega)]=\frac{1}{\pi} \operatorname{Im}[F] * \operatorname{coth}(\ln \omega)+\text { const, } \\
& \ln |F(\omega)|=\frac{1}{\pi} \arg [F] * \operatorname{coth}(\ln \omega)+\text { const. }
\end{aligned}
$$

In the MT-Corrector software (Nord-West Ltd.) used for post-processing and visualization of field MT data throughout the paper, the issue of the unknown constants is solved by automatic fitting of the calculated curves to the corresponding real part (log amplitude) spline.

By way of example, Fig. 8 shows the DR-II of both types as applied to the broadband MT (BBMT) data from site B-015 considered in Appendix 1. As seen from the figure, for the case when the phase is less biased than amplitude, the inverse DR-II provides a more intuitive insight of how the data should be corrected. At the same time, an easier-to-get but essentially equivalent forward DR-II apparently allows obtaining the same results, thus, for the sake of simplicity, below we will confine ourselves by considering only the dispersion relations of the forward type.

\section{Problems and limitations of the approach}

So far we tacitly meant that for ideal noise-free data the dispersion relations of both kinds must hold or, in other words, that the MT transfer functions under consideration are inherently causal and minimum-phase. Though 


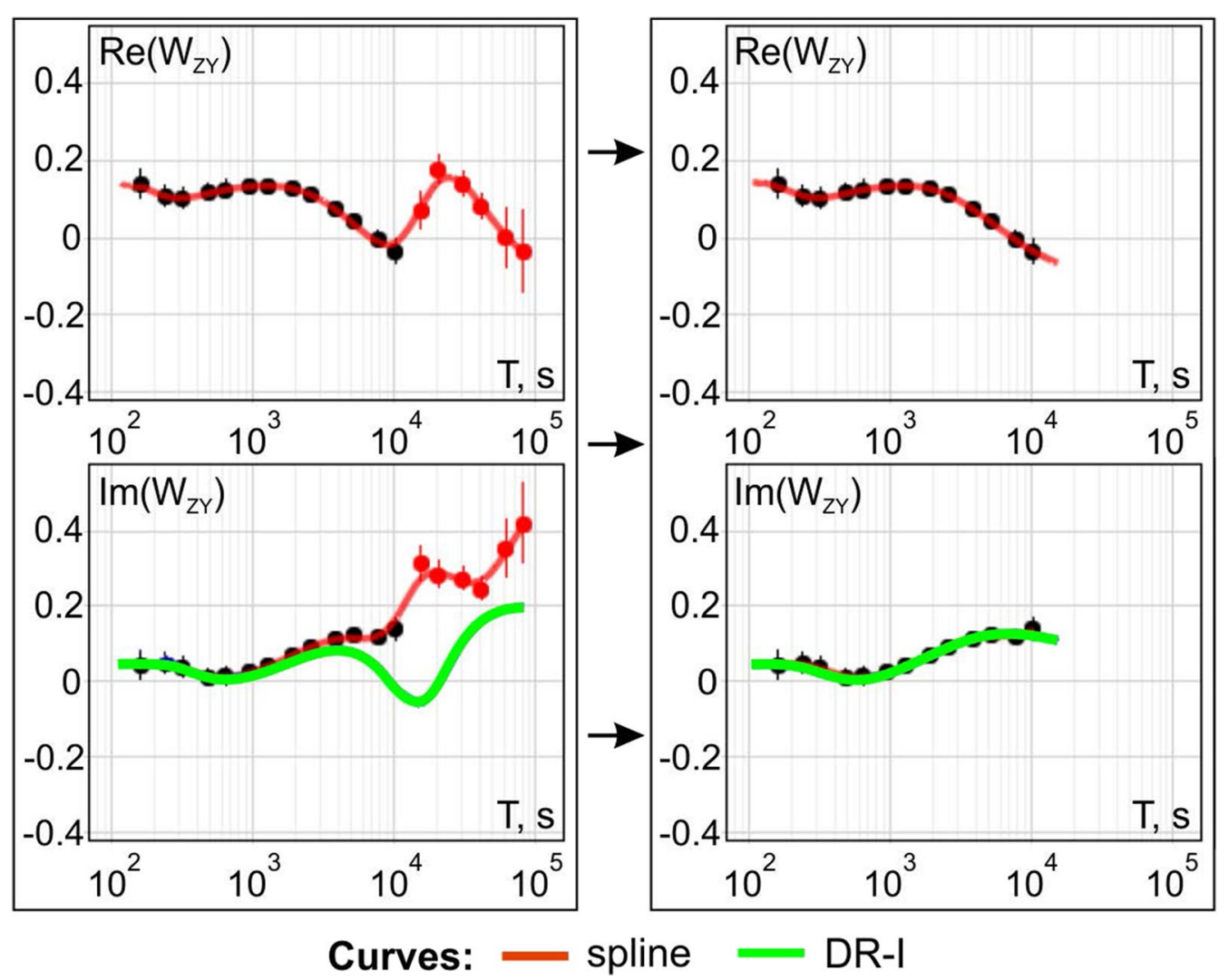

Fig. 7 Application of the DR-I to the tipper data acquired at CBI geomagnetic observatory in Chichijima, Japan; the data points distorted by Sq source effect are marked with red (modified after Shimizu et al. 2011)

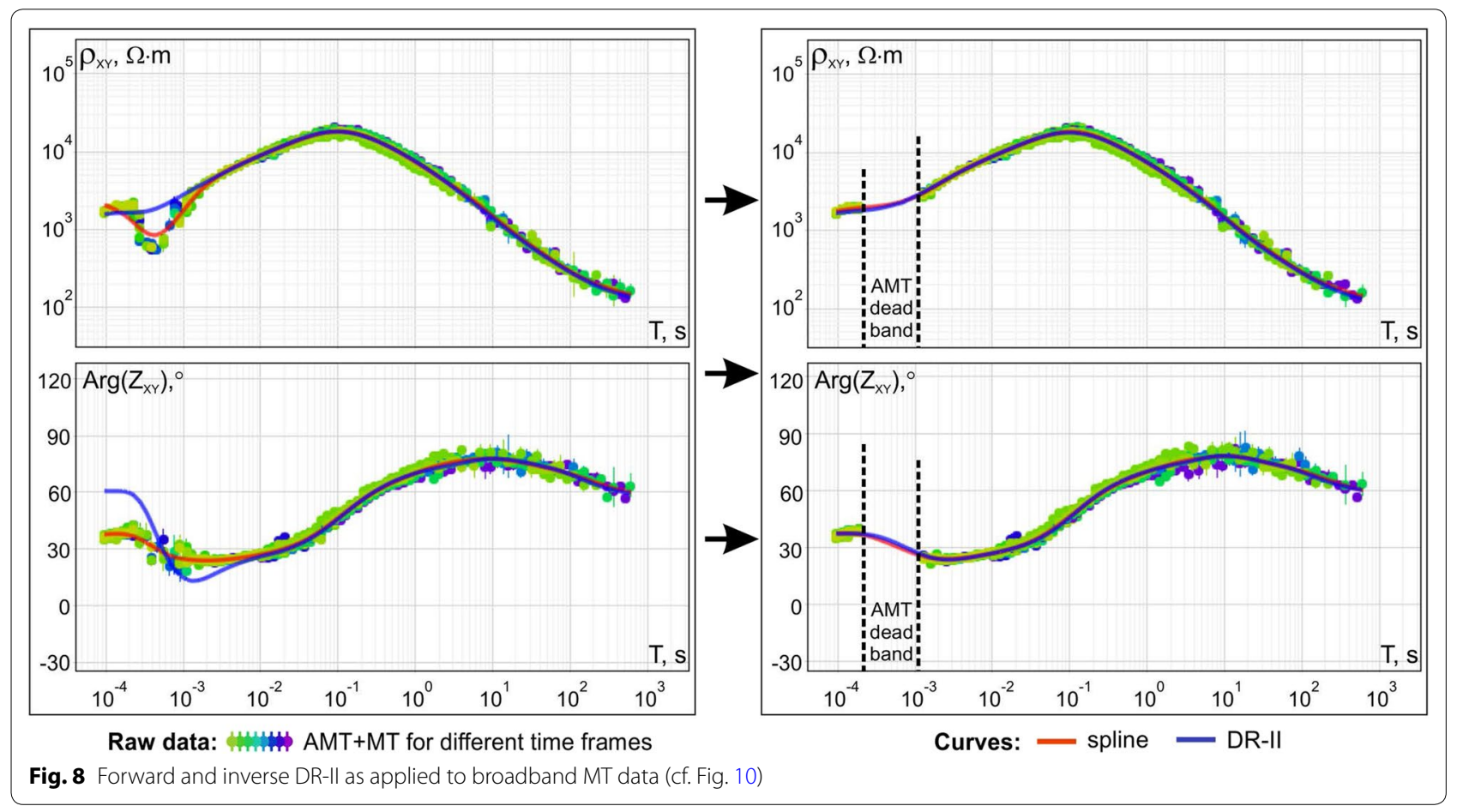


some authors (e.g., Yee and Paulson 1988, 1990) originally argued for the universal validity of these useful a priori assumptions, their unsupported statements have triggered a fierce controversy (see Egbert 1990; Svetov 1991; Berdichevsky 1999) as being at best nontrivial, and at worst-basically false.

An analytic proof of the DR validity exists for the MT impedance measured in isotropic 1-D medium (Weidelt 1972; see also Berdichevsky and Pokhotelov 1997; Weidelt 2003 for the case of frequency-dependent conductivity) and on the surface of isotropic $H$-polarized (magnetic field along the structures) 2-D medium with arbitrary topography (Weidelt and Kaikkonen 1994). Numerical simulations carried out by many researchers (Fischer and Schnegg 1993; Marcuello et al. 2005; Berdichevsky and Dmitriev 2008; Zorin and Alekseev 2018; etc.) suggest that MT impedance and tipper are minimum-phase on the surface of flat $E$-polarized (electric field along the structures) isotropic 2-D models as well. Finally, countless academic and commercial case studies demonstrate that the DR-II is applicable to the major portion of the apparent resistivity curves encountered on land.

At the same time, the MT observations also give a good deal of high-quality data examples with dramatic DR-II violation (Berdichevsky and Dmitriev 2008), most of them associated with the impedance phase "rolling out" of its quadrant (Chouteau and Tournerie 2000). In an attempt to explain the anomalous behavior of the observed transfer functions, the numerical simulations carried out in the last decades revealed at least three model classes where the DR-II could be violated. The first one requires the presence of a highly conductive 3-D body, whose shape may vary from a simple L-form to more exotic O-form, U-form, S-form and others (Berdichevsky 1999; Weckmann et al. 2003; Lezaeta and Haak 2003; Thiel et al. 2009; Ichihara and Mogi 2009; Kaufman et al. 2014). The second one consists of strongly anisotropic models (Heise and Pous 2003; Pek 2009; see also Marti 2014), and the last class considers the seafloor measurements in the presence of the coast effect, mostly described in MT literature by virtue of $E$ -polarized 2-D models (Alekseev et al. 2009; Kapinos and Brasse 2011; and others).

As things stand, today we have to admit that even if all the fundamental assumptions of the MT method (namely, those of the Earth being a linear, passive, timeinvariant system excited by a quasi-uniform source) are satisfied, the employed transfer functions do not generally have to be neither MP nor causal (Zorin and Alekseev 2018). This unfortunate fact significantly reduces the practical value of the DR application unless the following issues are appropriately addressed:
- How to distinguish between the DR violations due to noisy field data and those due to inherently non-MP/ non-causal behavior of the measured response?

- Could the DR still be applied to check the consistency and improve quality of non-MP/non-causal MT transfer functions?

The following section is intended to answer both of these principal questions.

\section{Validity of the DR in magnetotellurics} Non-minimum-phase functions

For solving the stated problems it is necessary to understand how the hypothetic appearance of lower half-plane zeros and/or poles in a given transfer function could generally affect the DR validity between its spectral components. The key point for such understanding lies in the concept of causal but non-MP functions considered below.

While the DR-I prescribes the existence of a unique causal function with given real part, there may exist any number of essentially different causal functions with the same amplitude. Among them, one can always distinguish a "canonical" (MP) function, whose phase and amplitude are related by the DR-II. All other transfer functions differ from the canonical one in that they have at least one zero in the lower half-plane. Each of these zeros leads to an additional positive phase shift. As a result, from all causal transfer functions with given amplitude, the DR-II formally highlights the one with minimum phase value (Toll 1956), which is responsible for the origin of the term "MP".

To demonstrate this, let us consider a causal but non-MP function $a_{n}$ with $n$ zeros in the lower half-plane, i.e., assume that for $k=\overline{1, n}$ there exist $\Omega_{k}=\left|\Omega_{k}\right| \exp \left(-i \alpha_{k}\right) \neq 0$ with $\alpha_{k} \in(0, \pi)$, such that $a_{n}\left(\Omega_{k}\right)=0$. Then we perform the canonical decomposition of $a_{n}$ by extracting all of its non-MP zeros without changing its amplitude as follows:

$$
a_{n}(\omega)=a_{o}(\omega) \prod_{k=1}^{n} \frac{\omega-\Omega_{k}}{\omega-\Omega_{k}^{*}}=a_{o}(\omega) b_{n}(\omega) .
$$

Since $\left|b_{n}\right| \equiv 1$, the derived function $a_{o}$ has the same amplitude as $a_{n}$ but no zeros in the lower half-plane, and hence is MP. By applying Eq. (2b) to Eq. (6), we may write the following relation between the spectral components of $a_{n}$ :

$$
\arg \left[a_{n}(\omega)\right]=\mathrm{DR}\left[\ln \left|a_{n}(\omega)\right|\right]+\theta_{n}(\omega),
$$

where $\theta_{n}$ is the phase of the $n$-component Blaschke product $b_{n}$ :

$$
\theta_{n}(\omega)=\arg \left[\prod_{k=1}^{n} \frac{\omega-\Omega_{k}}{\omega-\Omega_{k}^{*}}\right]=2 \sum_{k=1}^{n} \arg \left[\omega-\Omega_{k}\right] .
$$


Now it becomes clear that the amplitude and phase of an arbitrary causal function are in fact related by the generalized formula (7), which includes an unknown quantity $\theta \geq 0$ and, hence, is effectively an inequality (Bechhoefer 2011). For the present research it is important to note that, when applied to a causal but non-MP function $a_{n}$, the DR-II (Eq. 2b) turns out to be violated by the presence of an additional positive phase lag $\theta_{n}$. Let us consider its frequency behavior in detail.

According to the reflection symmetry property (Eq. 1), if $a_{n}$ has a single non-MP zero $(n=1)$, the latter is restricted to lie exactly on the negative imaginary axis, namely, at some point $\Omega_{1}=-i\left|\Omega_{1}\right|$. In this elementary case the non-MP term $\theta_{1}$ is given by

$$
\theta_{1}(\omega)=2 \arg \left[\omega+i\left|\Omega_{1}\right|\right]=2 \arctan \left[\exp \left(\ln \left|\Omega_{1}\right|-\ln \omega\right)\right] .
$$

This equation shows that on a log-period scale $\theta_{1}$ monotonically increases from 0 to $\pi$ and has the same, easily recognizable smooth shape, irrespective of the actual $\left|\Omega_{1}\right|$ value (Fig. 9a). Note that both $\omega$ and $z$ values at the plot are given in $\mathrm{rad} / \mathrm{s}$, hence the dotted semicircle $|\Omega|=2 \pi$ corresponds to the regular frequency value of $1 \mathrm{~Hz}$.

Let us consider a more complex case when $a_{n}(\Omega)$ has a conjugate pair of zeros $(n=2)$ located at the points $\Omega_{1}=\left|\Omega_{1}\right| \exp \left(-i \alpha_{1}\right) \quad$ and $\quad \Omega_{2}=\left|\Omega_{1}\right| \exp \left(i \alpha_{1}-i \pi\right)$, where $\alpha_{1} \in(0, \pi / 2]$. Substituting these values into Eq. (8) and making use of the properties of the inverse trigonometric and hyperbolic functions (Gradstein and Ryzhik 2007), we obtain

$$
\theta_{2}(\omega)=2 \arctan 2\left[\sin \alpha_{1}, \sinh \left(\ln \left|\Omega_{1}\right|-\ln \omega\right)\right],
$$

where $\arctan 2(y, x)$ is the 4-quadrant arctangent function returning the argument of a complex number $x+i y$ within the half-open interval $(-\pi, \pi]$. From Eq. (10) it follows that on a $\log$-period scale $\theta_{2}$ increases monotonically from 0 to $2 \pi$, while its shape varies from that of $2 \theta_{1}$ for $\alpha_{1}=\pi / 2$ (zeros coincide at the imaginary axis) to an abrupt $360^{\circ}$ flip for $\alpha_{1} \rightarrow 0$ (zeros approach the real axis), as shown at Fig. 9b. In the general case, an arbitrary phase lag $\theta_{n}$ may be represented as the corresponding sum of functions defined by either Eq. (9) or (10) and, hence, monotonically increases with period from 0 to $\pi n$.

The above results allow to make the following conclusions. First of all, we note that non-MP behavior should be considered as a local (in frequency) rather than global attribute of a given function. Indeed, though the DR-II in $a_{n}$ is formally violated almost everywhere along the frequency axis, the intensity of this violation is highly nonuniform. For instance, each of the non-MP lags depicted at Fig. 9 approaches zero at periods about $10^{-3} \mathrm{~s}$ and lower, hence the dispersion relation (Eq. $2 \mathrm{~b}$ ) between the amplitude and phase of $a_{n}$ turns out to be effectively valid everywhere within this interval. Furthermore, since the
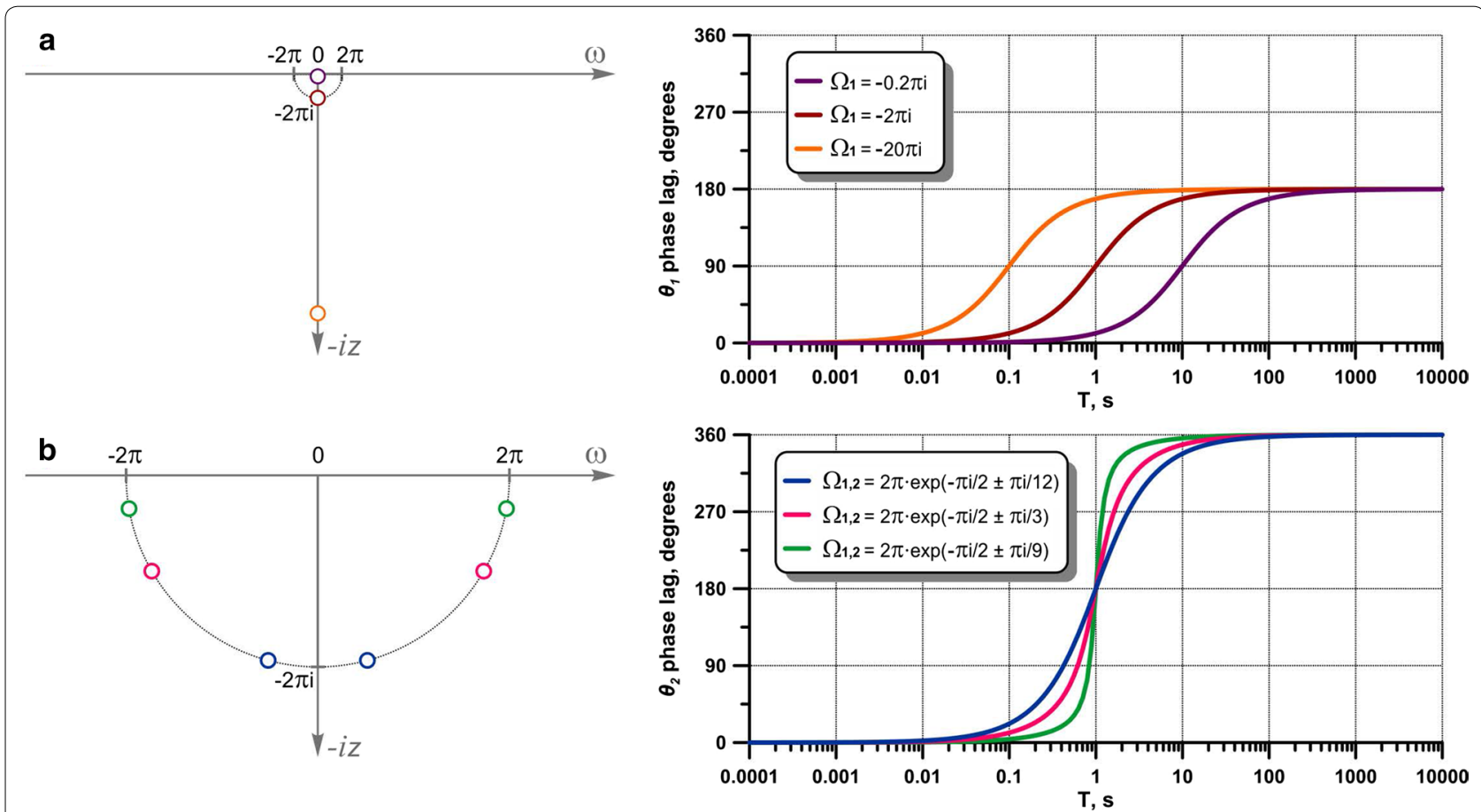

Fig. 9 Possible locations of non-MP zeros in the $\Omega$-plane (left) and the corresponding phase lags (right) due to: $\mathbf{a}$ a standalone zero; $\mathbf{b}$ a conjugate pair of zeros 
DR-II is intrinsically correct up to within an additive constant $\pi$, the same holds true at periods of about $10^{3} \mathrm{~s}$ and higher as well. Generally speaking, the DR-II violation caused by any zero located in a point $\Omega_{k}$ of the lower halfplane is ultimate around $\omega \sim\left|\Omega_{k}\right|$ and negligible for all frequencies $\omega \ll\left|\Omega_{k}\right|$ and $\omega \gg\left|\Omega_{k}\right|$. This means that the DR-II in its original form (Eq. 2b) is actually applicable not only to all MP functions, but also to those non-MP causal functions, which could be considered as "effectively MP" at the given frequency interval $\left[\omega_{1}, \omega_{2}\right]$-that is, to any function $a_{n}$, whose lower half-plane zeros $\Omega_{k}$ satisfy either of the conditions $\left|\Omega_{k}\right| \ll \omega_{1}$ or $\omega_{2} \ll\left|\Omega_{k}\right|$ for each $k=\overline{1, n}$.

Now, let us assume that the zeros of $a_{n}$ correspond to the measured frequency range. Though this situation would force the DR-II in $a_{n}$ spectrum to be considerably violated by a presence of an unknown phase lag $\theta_{n}$ (Eq. 8), the latter must belong to a very specific and easily recognizable family of curves shown in Fig. 9. This means that the DR-II may be applied to the effectively non-MP causal functions as well-for that one just needs to correctly identify the type of the observed non-MP lag $\theta_{n}$, define its order $n$ (which is equal to the number of "unexpected" $180^{\circ}$ phase flips) and then make use of the generalized DR-II (Eq. 7) with $n$ additional unknowns instead of Eq. (2b).

An attentive reader must have already noticed the striking similarity between the phase curves given in Fig. 9 and many examples of non-MP data revealed by both MT modeling and practice. Running slightly ahead, we may say that, indeed, the synthetic impedances with rolling-out-of-quadrant phases discussed in, e.g., Berdichevsky (1999), Heise and Pous (2003), Ichihara and Mogi (2009), Pek (2009), as well as the vast majority of similar MT data observed in the field, are causal transfer functions with just one or two non-MP zeros. This makes possible to solve the whole issue of the correct DR application to such data merely by using the DR-I (Eq. 2a) or generalized DR-II (Eq. 7) instead of the regular DR-II (Eq. 2b).

\section{Causality of magnetotelluric transfer functions}

Prior to proceeding further, we need to briefly list some additional properties of the causal and minimum-phase functions on the $\Omega$-plane. Firstly, a product of two causal (or minimum-phase) functions is also a causal (or MP) function. Secondly, a linear combination of causal functions is causal, while such a combination of MP functions could be either minimum-phase or not. Finally, the ratio of two causal functions is universally causal only if the function in the denominator is MP (otherwise any lower half-plane zero of the latter would become a potential lower half-plane pole of the whole ratio).
Another important thing to be taken into account is that the MT method works with tensor functions (Berdichevsky and Dmitriev 2008). Since conversion of these functions from one orthonormal basis to another is a linear transformation, the causal behavior of their components observed in any given coordinate system would be preserved for all other coordinate systems as well, and hence could be considered as an intrinsic property of the whole tensor. This allows introducing the following practical definition: we would call a Cartesian tensor "causal" if all its components are causal functions, and "non-causal" if this is not the case. Notably, the same definition could not be extended for the (conceptual) minimum-phase property of a tensor, since its validity would depend on the employed coordinate system.

Consider the Earth to be a linear (with respect to the source field), passive, time-invariant system excited by planar quasi-uniform source located far in the non-conducting upper half-space. Then the external source field $\boldsymbol{G}$ is related to the horizontal electric $\boldsymbol{E}$ and magnetic $\boldsymbol{H}$ field components observed on the surface or inside the conductive Earth as follows (hereafter the dependence on $\omega$ is omitted in equations for simplicity):

$$
\begin{gathered}
\boldsymbol{E}=\left[\begin{array}{l}
E_{x} \\
E_{y}
\end{array}\right]=\left[\begin{array}{ll}
e_{x x} & e_{x y} \\
e_{y x} & e_{y y}
\end{array}\right]\left[\begin{array}{l}
G_{x} \\
G_{y}
\end{array}\right]=\hat{e} \boldsymbol{G}, \\
\boldsymbol{H}=\left[\begin{array}{l}
H_{x} \\
H_{y}
\end{array}\right]=\left[\begin{array}{ll}
h_{x x} & h_{x y} \\
h_{y x} & h_{y y}
\end{array}\right]\left[\begin{array}{l}
G_{x} \\
G_{y}
\end{array}\right]=\hat{h} \boldsymbol{G} .
\end{gathered}
$$

The "horizontal electric field tensor" $\hat{e}$ and "horizontal magnetic field tensor" $\hat{h}$ describe the respective responses of the black-box Earth to an external cause $\boldsymbol{G}$ and, hence, are causal by definition. Though for synthetic MT models these tensors could be estimated either analytically or numerically, they could never be derived from the field data due to unknown characteristics of the real input signal. As a result, the magnetotelluric method is limited to dealing with "mutual" transfer functions, which relate two output signals and hence are not necessarily causal (Egbert and Booker 1989; Svetov 1991). The basic MT function is the impedance tensor $\hat{Z}$ defined by:

$$
\boldsymbol{E}=\left[\begin{array}{c}
E_{x} \\
E_{y}
\end{array}\right]=\left[\begin{array}{ll}
Z_{x x} & Z_{x y} \\
Z_{y x} & Z_{y y}
\end{array}\right]\left[\begin{array}{l}
H_{x} \\
H_{y}
\end{array}\right]=\hat{Z} \boldsymbol{H}
$$

Comparing Eq. (12) and Eq. (11), we get $\hat{e}=\hat{Z} \hat{h}$, hence $\hat{Z}=\hat{e} \hat{h}^{-1}$, or

$$
\left[\begin{array}{ll}
Z_{x x} & Z_{x y} \\
Z_{y x} & Z_{y y}
\end{array}\right]=\frac{1}{\operatorname{det} \hat{h}}\left[\begin{array}{ll}
e_{x x} & e_{x y} \\
e_{y x} & e_{y y}
\end{array}\right]\left[\begin{array}{cc}
h_{y y} & -h_{x y} \\
-h_{y x} & h_{x x}
\end{array}\right],
$$

where $\operatorname{det} \hat{h}=\left(h_{x x} h_{y y}-h_{x y} h_{y x}\right)$ is the $\hat{h}$ matrix determinant. This equation indicates that if the inherently causal 
function $\operatorname{det} \hat{h}$ is also minimum-phase, the impedance tensor $\hat{Z}$ has to be causal and, conversely, non-causal behavior of $\hat{Z}$ is possible only if $\operatorname{det} \hat{h}$ is non-MP. Applying similar approach to the tipper $\hat{W}$

$$
H_{z}=\left[W_{z x} W_{z y}\right]\left[\begin{array}{c}
H_{x} \\
H_{y}
\end{array}\right]=\hat{W} \boldsymbol{H},
$$

it is straightforward to show that the conditions of its causality are exactly the same. This particular observation appears to be of high practical importance, since it shows that at every MT survey site characterized by causal behavior of the impedance tensor, the tipper components must also be causal.

Alternatively, if we consider magnetic tensor $\hat{M}$

$$
\boldsymbol{H}=\left[\begin{array}{l}
H_{x} \\
H_{y}
\end{array}\right]=\left[\begin{array}{ll}
M_{x x} & M_{x y} \\
M_{y x} & M_{y y}
\end{array}\right]\left[\begin{array}{l}
H_{x}^{\text {base }} \\
H_{y}^{\text {base }}
\end{array}\right]=\hat{M} \boldsymbol{H}^{\text {base }},
$$

inter-site impedance tensor $\hat{Q}$ (Kruglyakov and Kuvshinov 2019)

$$
\boldsymbol{E}=\left[\begin{array}{c}
E_{x} \\
E_{y}
\end{array}\right]=\left[\begin{array}{ll}
Q_{x x} & Q_{x y} \\
Q_{y x} & Q_{y y}
\end{array}\right]\left[\begin{array}{c}
H_{x}^{\text {base }} \\
H_{y}^{\text {base }}
\end{array}\right]=\hat{Q} \boldsymbol{H}^{\text {base }},
$$

inter-site tipper (Schmucker matrix) $\hat{S}$ (Berdichevsky and Dmitriev 2008)

$$
H_{z}=\left[S_{z x} S_{z y}\right]\left[\begin{array}{c}
H_{x}^{\text {base }} \\
H_{y}^{\text {base }}
\end{array}\right]=\hat{S} \boldsymbol{H}^{\text {base }},
$$

or any other transfer function using the horizontal magnetic field $\boldsymbol{H}^{\text {base }}$ from a remote base site instead of $\boldsymbol{H}$ as an "input", the key role in the subject of their causality would be naturally transferred from $\operatorname{det} \hat{h}$ to $\operatorname{det} \hat{h}^{\text {base }}$.

As an equivalent alternative to $\hat{Z}$, one may also choose to employ the admittance tensor $\hat{Y}$ (Berdichevsky and Dmitriev 2008), defined by:

$$
\boldsymbol{H}=\left[\begin{array}{l}
H_{x} \\
H_{y}
\end{array}\right]=\left[\begin{array}{cc}
Y_{x x} & Y_{x y} \\
Y_{y x} & Y_{y y}
\end{array}\right]\left[\begin{array}{c}
E_{x} \\
E_{y}
\end{array}\right]=\hat{Y} \boldsymbol{E} .
$$

While the impedance function transforms $\boldsymbol{H}$ into $\boldsymbol{E}$, the admittance does just the opposite, treating the electric field as a formal input. Consequently, the causality of $\hat{Y}$ does not depend on the magnetic field behavior, being fully governed by the local electric response det $\hat{e}$. Indeed, comparing Eq. (18) and Eq. (12), we get $\hat{Y}=\hat{Z}^{-1}=\hat{h} \hat{e}^{-1}$, or

$$
\left[\begin{array}{cc}
Y_{x x} & Y_{x y} \\
Y_{y x} & Y_{y y}
\end{array}\right]=\frac{1}{\operatorname{det} \hat{e}}\left[\begin{array}{ll}
h_{x x} & h_{x y} \\
h_{y x} & h_{y y}
\end{array}\right]\left[\begin{array}{cc}
e_{y y} & -e_{x y} \\
-e_{y x} & e_{x x}
\end{array}\right]
$$

which makes for another useful observation that the admittance tensor could generally be causal even when the impedance tensor at the same MT site is not.
Finally, for the telluric tensor $\hat{T}$

$$
\boldsymbol{E}=\left[\begin{array}{c}
E_{x} \\
E_{y}
\end{array}\right]=\left[\begin{array}{ll}
T_{x x} & T_{x y} \\
T_{y x} & T_{y y}
\end{array}\right]\left[\begin{array}{c}
E_{x}^{\text {base }} \\
E_{x}^{\text {base }}
\end{array}\right]=\hat{T} \boldsymbol{E}^{\text {base }},
$$

and any other transfer function using the electric field $E^{\text {base }}$ from a remote base site as an "input", the key role in the subject of causality is further transferred to det $\hat{e}^{\text {base }}$.

From above we conclude that one should expect causal behavior to be the rule rather than exception not only for $Z_{x y}$ and $Z_{y x}$, but, in fact, for all components of $\hat{Z}, \hat{W}$ and any other MT tensor which employs horizontal magnetic field as a formal input. Furthermore, even if some local tensor $\hat{X}$ happens to be non-causal, the DR could yet be applicable to its inverse $\hat{X}^{-1}$. Finally, in the unlikeliest but theoretically possible situation when both $\hat{X}$ and $\hat{X}^{-1}$ are non-causal, the causality would still be preserved for any inter-site transfer function measured at the same location, provided that the base site is chosen in relatively "calm" area with regular MT field behavior. In other words, this result suggests that by careful choice of the appropriate target function and kind of relation (Eqs. 2a, 2b or 7) the DR applicability may be extended to the data obtained virtually at any survey location.

In order to translate the proposed approach into practice, it is necessary to know how to distinguish between a DR violation caused by noisy field data and that being an intrinsic attribute of the measured (nonMP or non-causal) response. This issue is discussed in the following.

\section{Three classes of the DR validity}

For brevity, in this subsection we will confine ourselves to consideration of the impedance tensor, which happens to be the most popular transfer function of the MT method. However, all the reasoning and conclusions given below could be easily repeated for the other MT tensors as well.

As shown by Eq. (13), any impedance component $Z_{i j}$ in any given coordinate system may be represented as the ratio of two causal (but not necessarily MP) functions $d_{i j}$ and $\operatorname{det} \hat{h}$ :

$$
Z_{i j}=\frac{d_{i j}}{\operatorname{det} \hat{h}} .
$$

From the standpoint of the DR validity this suggests the existence of four principal situations:

1. Both $d_{i j}$ and $\operatorname{det} \hat{h}$ are MP.

2. Only det $\hat{h}$ is MP.

3. Only $d_{i j}$ is MP.

4. Neither $d_{i j}$ nor $\operatorname{det} \hat{h}$ is MP. 
The 1st case is trivial, since $Z_{i j}$ is a MP function and the dispersion relations of both kinds between its spectral components are valid for all frequencies $\omega$.

In the 2nd case $Z_{i j}$ turns out to be a causal but non-MP function. As shown above, this fact alone does not necessarily imply a detectable DR-II violation within the measured frequency range $\left[\omega_{1}, \omega_{2}\right]$, since $Z_{i j}$ may be effectively MP on this interval. At the same time, if at least one nonMP zero of $Z_{i j}$ corresponds to the measured frequency range, we should expect the DR-II to be violated in a very specific and easily recognizable way (Fig. 9). In this situation one may choose between using the DR-I (Eq. 2a), the generalized DR-II (Eq. 7) with $n$ unknown constants, or try rotating the coordinate system in order to get rid of the non-MP zeros and then apply the regular DR-II (Eq. 2b) instead. Though some difficulties in recognizing non-MP behavior could be faced if a complex plane zero of $Z_{i j}$ is found close to some real frequency $\omega_{0} \in\left[\omega_{1}, \omega_{2}\right]$, this would also require that $\ln \left|Z_{i j}\right|$ curve would have a characteristic "negative cusp" (sharp peak pointing down) at $\omega \approx \omega_{0}$, and application of the DR-I or slight rotation of the impedance tensor be preferable anyway.

In the 3rd case $Z_{i j}$ is non-causal, while its reciprocal $Z_{i j}^{-1}=1 / Z_{i j}$ belongs to the class of causal non-MP functions described above. As a consequence, if all the lower half-plane poles of $Z_{i j}$ are located far from measured frequencies, both $Z_{i j}^{-1}$ and $Z_{i j}$ would be effectively MP on the interval, and both dispersion relations in $Z_{i j}$ be valid. This result implies that not only non-MP, but also non-causal behavior of an MT transfer function should be regarded as its local (in frequency) rather than global attribute. At the same time, if $m$ non-causal poles of $Z_{i j}$ correspond to the measured frequency range, we should expect the DR-II be violated exactly by the amount of $-\theta_{m}$ (i.e., non-causal phase lags are inherently negative), and the DR-I "predicting" incorrect sign for the actual $\operatorname{Im}\left[Z_{i j}\right]$ values (Appendix 2). In this situation, for proper use of the dispersion relations, one may consider their application to the admittance tensor $\hat{Y}=\hat{Z}^{-1}$ or the corresponding inter-site transfer functions (e.g., $\hat{M}$ and $\hat{Q}=\hat{M} \hat{Z}$ ) instead of $\hat{Z}$. Again, though some difficulties in recognizing non-causal behavior of $Z_{i j}$ could be faced if one of its complex plane poles is found close to a real frequency $\omega_{0} \in\left[\omega_{1}, \omega_{2}\right]$, this would also initiate a positive cusp of $\ln \left|Z_{i j}\right|$ at $\omega \approx \omega_{0}$, and consideration of the admittance tensor $Y$ or the corresponding inter-site transfer functions instead of $\hat{Z}$ would be preferable anyway.

The 4th case represents a combination of the 2nd and the 3rd ones and, hence, appears to be the worst from the standpoint of its practical identifiability. Indeed, if $Z_{i j}$ has both poles and zeros in the lower half-plane, the associated negative and positive phase lags sum up, and the corresponding DR-II violation may lose its characteristic shape depicted in Fig. 9. Furthermore, if a pole and a zero of the same order are located close to some point $\Omega^{\prime}$ of the lower-half plane, their phase lags would largely compensate each other, resulting in only a minor DR-II violation at frequencies $\omega \approx\left|\Omega^{\prime}\right|$. As a consequence, a reliable identification of a non-causal tensor may require examination of all its components together. An effective tool for confirmation of non-causal behavior for a "suspicious" tensor is gradual rotation of the coordinate system. Indeed, since this procedure generally affects $d_{i j}$ without changing $\operatorname{det} \hat{h}$, it forces the zeros of $Z_{i j}$ to move along the $\Omega$-plane, while all the $Z_{i j}$ poles retain their original position, thus making the DR-II violation more (or less) recognizable.

The obtained results allow us to introduce three general classes of MT transfer functions:

- class 1-MP components of a causal tensor;

- class 2-non-MP components of a causal tensor;

- class 3-all components of a non-causal tensor.

As applied to an impedance tensor component $Z_{i j}$, these 3 classes are described in detail in Table 1, which makes it possible to distinguish the intrinsic DR violations from those caused by noisy data, and appropriately apply one or another dispersion relation to the measured response. Formulation of similar tables for the other MT transfer functions is straightforward. Note that, if the harmonic time factor $e^{-i \omega t}$ is used instead of $e^{i \omega t}$, all the observed phases would have inverse signs and hence demonstrate exactly opposite frequency behavior. A universal and simple mnemonic rule is that on log-period scale the non-MP phase lags drive the phase of $Z_{x y}$ originating in its regular quadrant away from $0^{\circ}$ value, while non-causal-towards (and across) it.

\section{Summary}

The principal result of our research is that the DR applicability to MT data happens to be of much more universal nature than it is generally believed. Indeed, the dispersion relations of both kinds are in fact applicable to any component of any causal MT tensor: the DR-I in its original form (Eq. 2a), and the DR-II-either in its original (Eq. 2b) or generalized (Eq. 7 with $n \neq 0$ ) forms, where the latter applies only to the functions with out-of-quadrant phase values. Moreover, even if a tensor in hand happens to be non-causal, it may be equivalently represented via some other causal tensors from the given response space. As a consequence, the dispersion relations could be applied virtually to any MT data set by making use of the appropriate target function and kind of relation. A key point for success 
Table 1 Classification of the impedance tensor components from the standpoint of the DR validity

\begin{tabular}{|c|c|c|c|}
\hline$Z_{i j}$ & Class 1 & Class 2 & Class 3 \\
\hline$\hat{Z}$ causality & Causal & Causal & Non-causal \\
\hline DR-II validity & Valid & $\begin{array}{l}\text { Violated by the amount of a positive } \\
\text { monotonic phase lag } \theta_{n} \text { (Eq. 8) }\end{array}$ & $\begin{array}{l}\text { Violated by the amount of a negative monotonic phase lag }-\theta_{m} \text {, } \\
\text { or a non-monotonic phase lag } \theta_{n}-\theta_{m} \text { (Eq. 8) }\end{array}$ \\
\hline DR-I validity & Valid & Valid & Invalid (unless $\theta_{n} \equiv \theta_{m}$ ) \\
\hline $\begin{array}{l}\text { Characteristic features of } \\
\arg \left[Z_{i j}\right] \text { on log-period scale }\end{array}$ & - & $\begin{array}{l}\text { Rolls out of its quadrant upwards and/or } \\
\text { rolls in from below }\end{array}$ & $\begin{array}{l}\text { Rolls out of its quadrant downwards and/or rolls in from above at } \\
\text { least at some angle of } \hat{Z} \text { rotation }\end{array}$ \\
\hline Typical features of $\ln \left|Z_{i j}\right|$ & - & May reveal a negative cusp if $\hat{Z}$ is rotated & May reveal a cusp if $\hat{Z}$ is rotated \\
\hline How the DR-II could be applied & Directly & By using Eq. (7) with $n$ unknowns & \multirow{2}{*}{$\begin{array}{l}\text { Consider the DR application to } \hat{Y}=\hat{Z}^{-1} \text { or the corresponding } \\
\text { inter-site tensors instead }\end{array}$} \\
\hline How the DR-I could be applied & & & \\
\hline
\end{tabular}

Class 1-refers to MP components of a causal tensor, class 2 - to non-MP components of a causal tensor, and class 3-to all components of a non-causal tensor

of this procedure is to correctly classify the considered transfer functions in terms of the DR validity (Table 1).

Illustrations of the above theoretical conclusions on synthetic and field data examples are given in the second part of the paper, which is also intended to finally answer the old but principal question raised in the controversy between Egbert (1990) and Yee and Paulson (1988, 1990): could a non-causal impedance tensor be actually encountered in geologically reasonable models and, hence, in real MT exploration?

\section{Supplementary information}

Supplementary information accompanies this paper at https://doi. org/10.1186/s40623-020-1133-4.

Additional file 1. Field data pack.

\section{Abbreviations}

MT: magnetotelluric; AMT: audio-frequency magnetotelluric; BBMT: broadband (AMT + MT) magnetotelluric; DR: dispersion relation; DR-I: dispersion relation of the first kind; DR-II: dispersion relation of the second kind; MP: minimumphase; DC: direct current; 1-D: one-dimensional; 2-D: two-dimensional; 3-D: three-dimensional.

\section{Acknowledgements}

The tipper responses distorted by Pc3-4 source effect were kindly provided by Dr. B. Murphy. We thank Dr. A. Kuvshinov for responsible editorial handling and two anonymous reviewers for their thorough reading of the manuscript and helpful remarks. We also would like to express deep gratitude to Drs. A Yakovlev, N. Palshin, H. Utada and many other members of Nord-West Ltd. and Earthquake Research Institute, without whom this collaborative research project could not be realized.

\section{Authors' contributions}

$\mathrm{NZ}$ and HS (corresponding author) —writing and compiling the manuscript. NZ_-theory. EA, HS and DY_ provision and selection of the field data. DYpreparation of figures. All authors read and approved the final manuscript.

\section{Funding}

The research stays of NZ at the University of Tokyo were funded by ShortTerm Postdoctoral Fellowship for Research in Japan in FY 2018-2019 (JSPS/ OF165) from the Japan Society for the Promotion of Science (JSPS), and by Students and Researchers Exchange Program (STEPS) in FY 2017 from the Ministry of Education, Culture, Sports, Science and Technology (MEXT). HS was supported by Grants-in-Aid for Scientific Research (KAKENHI) JP18H01302 and JP18H03735 from the Japan Society for the Promotion of Science (JSPS).

Availability of data and materials

The MT responses referred to in Figs. 5, 8 and 10 are included as additional file.

Ethics approval and consent to participate

Not applicable.

Consent for publication

Not applicable.

Competing interests

The authors declare that they have no competing interests.

Author details

${ }^{1}$ Nord-West Ltd., Business Centre Rumyantsevo, 1A, Office 412A, Moscow 108811, Russia. ${ }^{2}$ Earthquake Research Institute, The University of Tokyo, 1-1-1, Yayoi, Bunkyo-ku, Tokyo 113-0032, Japan.

\section{Appendix}

\section{Appendix 1: Post-processing of MT data}

In this appendix we consider a detailed example of the post-processing routine as applied to a highly noise-contaminated broadband MT (BBMT) measurement.

A 2-day BBMT record B-015 (Additional file 1) was taken in June, 2015, during regional investigations in Buryatia, Southeastern Russia. The measurement consists of two separate parts recorded by different sets of MTU equipment (Phoenix Geophysics Ltd.)-one with audio-frequency magnetic coils AMTC-30 (brown dots at Fig. 10a), and another one with low-frequency coils MTC-50 (black dots). The survey site is located less than $10 \mathrm{~km}$ away of Irokinda gold mine, which caused an extensive contamination of the observed data with industrial noise of predominantly low-frequency nature. Though at a first glance the processed data may seem to have rather poor or even unsatisfactory quality, in fact they allow obtaining reliable and well-defined values of the impedance function for all 7 decades of frequency, which could be achieved as follows. 

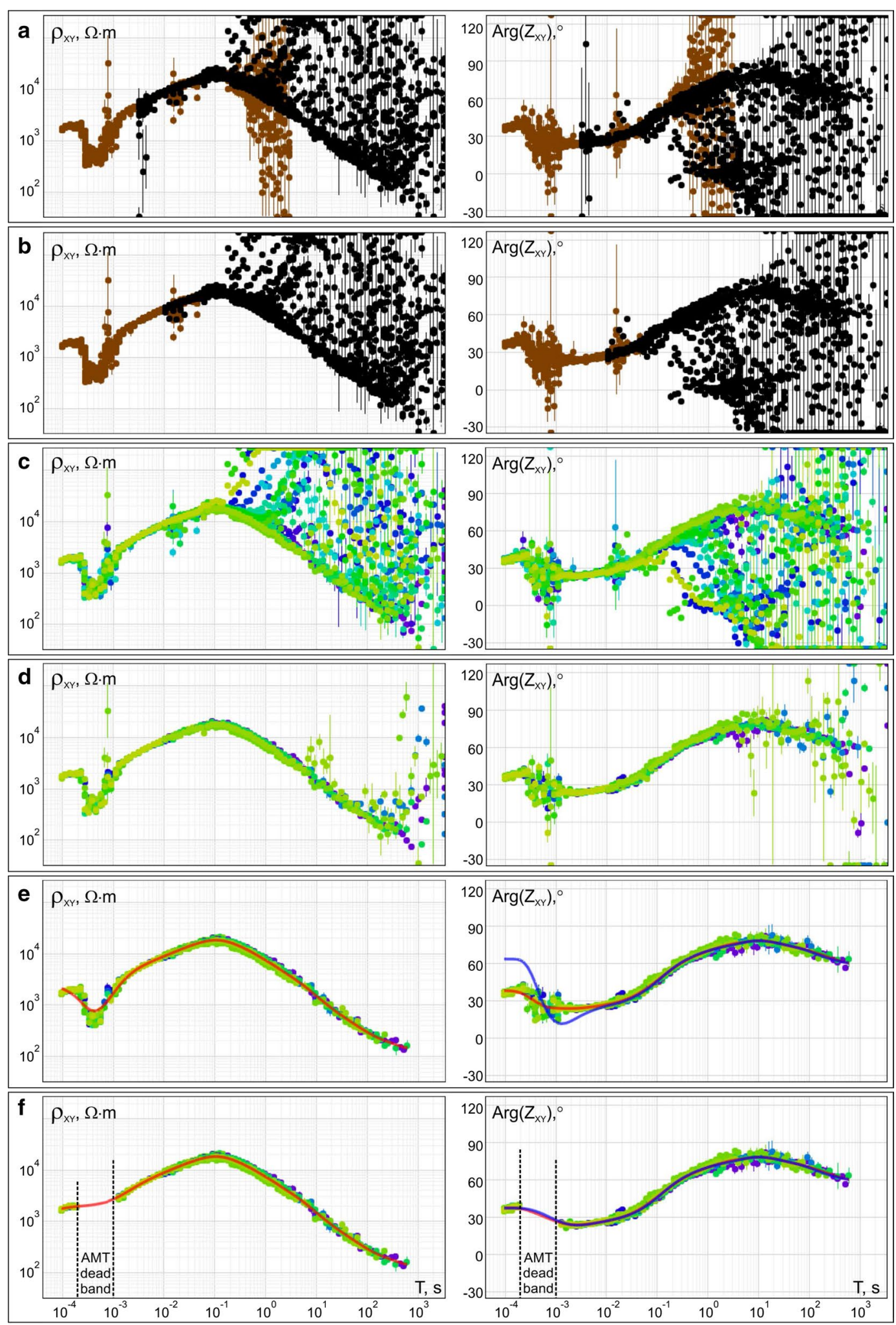

Raw data: $\phi \phi$ AMT $\phi \phi \phi M T$ AMT+MT for different time frames Curves: - spline - DR-II

Fig. 10 Post-processing of a noisy BBMT record acquired in Buryatia, Southeastern Russia: $\mathbf{a}$, $\mathbf{b}$ removal of the estimations obtained at frequencies characterized by increased sensor noise; $\mathbf{c}$, $\mathbf{d}$ removal of the estimations obtained for the time frames (marked with different colors) characterized by high data scatter; $\mathbf{e}$, f removal of the outliers and biased data to get smooth curves consistent with the DR-II 
The first step of the procedure is to remove data distorted by instrumental noise. Since the optimal operating frequency bands of the employed magnetic coils overlap within the region of approximately $3-100 \mathrm{~Hz}\left(10^{-2}\right.$ $s-3 \times 10^{-1} \mathrm{~s}$ ), the estimations obtained at frequencies characterized by increased sensor noise (those higher than $100 \mathrm{~Hz}$ for MT data and lower than $3 \mathrm{~Hz}$ for AMT data) and hence increased scatter, could be removed (Fig. 10b). The next step is intended to make advantage of the fact that though the underground mining and ore milling procedures are carried out $24 / 7$, there still must be shift changes and other short brakes characterized by drastically reduced level of industrial noise. For that purpose the estimations obtained within each of 20 time frames employed in data processing were marked with different colors (Fig. 10c) and 5 time frames with the lowest estimation scatter were chosen for further consideration (Fig. 10d). This procedure significantly improved the data quality around the power-line frequency $(50 \mathrm{~Hz})$ and at longer periods, making removal of the remaining outliers and application of spline approximation routine in the next step (Fig. 10e) much easier. Involvement of the DR into the latter step helps to determine the inconsistent (e.g., substantially biased) data estimations. A common reason for the bias to appear in the results of automatic MT processing is low signal-to-noise ratio (Chave 2012), which is often observed at frequencies within the "dead bands", characterized by reduced values of the natural-source power spectrum (Simpson and Bahr 2005). The AMT dead band is known to be at frequencies about $1-5 \mathrm{kHz}$ (Viljanen 2012), which serves as a plausible explanation of the discrepancy between the DR-II curve and corresponding phase data in the left part of Fig. 10e. Since the phase estimations are generally less biased by random noise in raw MT data, sometimes it is sufficient to correct only the amplitude (Boehl et al. 1977). However, if the data outside the dead band are accurate, one may even remove estimations for both components, since their actual behavior inside the dead band could be fully recovered using only the DR-II and smoothness constraints (Fig. 10f).

\section{Appendix 2: DR-I violation in non-causal functions}

Here we shall derive some approximate expressions describing the principal behavior of the DR-I violation in a non-causal transfer function $A_{m}$ :

$$
A_{m}=\operatorname{Re}\left[A_{m}\right]+i \operatorname{Im}\left[A_{m}\right]=\left|A_{m}\right| \cdot\left(\cos \varphi_{m}+i \sin \varphi_{m}\right),
$$

which is free of zeros, but has $m$ poles in the lower half of the complex frequency plane.

Since $A_{m}$ is free of zeros in the lower half-plane, its reciprocal $A_{m}^{-1}=1 / A_{m}$ is a causal function with real $\operatorname{Re}\left[A_{m}^{-1}\right]=\operatorname{Re}\left[A_{m}\right] /\left|A_{m}\right|^{2}$ and imaginary
$\operatorname{Im}\left[A_{m}^{-1}\right]=-\operatorname{Im}\left[A_{m}\right] /\left|A_{m}\right|^{2}$ parts related by the DR-I (Eq. 2a):

$$
-\frac{\operatorname{Im}\left[A_{m}\right]}{\left|A_{m}\right|^{2}}=\operatorname{DR}\left[\frac{\operatorname{Re}\left[A_{m}\right]}{\left|A_{m}\right|^{2}}\right] .
$$

At the same time, $A_{m}^{-1}$ has $m$ zeros in the lower halfplane, hence, its amplitude $\left|A_{m}^{-1}\right|=1 /\left|A_{m}\right|$ and phase $\arg \left[A_{m}^{-1}\right]=-\varphi_{m}$ are related by the generalized DR-II (Eq. 7):

$$
-\varphi_{m}=-\beta_{m}+\theta_{m}
$$

where $\beta_{m}=\mathrm{DR}\left[\ln \left|A_{m}\right|\right]$, and $\theta_{m}$ is the phase angle of the $m$-component all-pass Blaschke product given by Eq. (8).

Having Eq. (22) and Eq. (23) in hand, we want to estimate the real-valued parameter $p$ in the relation:

$$
\mathrm{DR}\left[\operatorname{Re}\left[A_{m}\right]\right]=p \cdot \operatorname{Im}\left[A_{m}\right],
$$

which would characterize the DR-I violation in $A_{m}$. For this purpose, we make use of the simplified (approximate) form of the DR (e.g., Weidelt 1972; Boehl et al. 1977):

$$
\operatorname{DR}[y] \approx \frac{\pi}{2} \frac{d y}{d \ln \omega}
$$

In this case Eq. (22) may be first rewritten as

$$
\begin{aligned}
-\frac{\operatorname{Im}\left[A_{m}\right]}{\left|A_{m}\right|^{2}} \approx & \frac{\pi}{2} \cdot \frac{1}{\left|A_{m}\right|^{4}} \\
& \left(\frac{d \operatorname{Re}\left[A_{m}\right]}{d \ln \omega}\left|A_{m}\right|^{2}-2\left|A_{m}\right| \frac{d\left|A_{m}\right|}{d \ln \omega} \operatorname{Re}\left[A_{m}\right]\right),
\end{aligned}
$$

and then as

$$
-\operatorname{Im}\left[A_{m}\right] \approx \mathrm{DR}\left[\operatorname{Re}\left[A_{m}\right]\right]-2 \operatorname{Re}\left[A_{m}\right] \beta_{m} .
$$

Combination of Eq. (25) with Eq. (24) yields:

$$
-\operatorname{Im}\left[A_{m}\right] \approx p \cdot \operatorname{Im}\left[A_{m}\right]-2 \operatorname{Re}\left[A_{m}\right] \beta_{m},
$$

or, after dividing both parts by $\left|A_{m}\right|$ :

$$
p \sin \varphi_{m} \approx 2 \beta_{m} \cos \varphi_{m}-\sin \varphi_{m} .
$$

Then, making an additional assumption that $\beta_{m}$ is small enough to consider $\sin 2 \beta_{m} \approx 2 \beta_{m}$ and $\cos 2 \beta_{m} \approx 1$, we get

$$
p \sin \varphi_{m} \approx \sin 2 \beta_{m} \cos \varphi_{m}-\cos 2 \beta_{m} \sin \varphi_{m},
$$

or

$$
p \sin \varphi_{m} \approx \sin \left(2 \beta_{m}-\varphi_{m}\right)
$$

Finally, substitution of Eq. (23) into Eq. (26) yields: 


$$
p \approx \frac{\sin \left(\beta_{m}+\theta_{m}\right)}{\sin \left(\beta_{m}-\theta_{m}\right)} .
$$

The obtained result expectedly shows that $p \approx 1$ for $\theta_{m} \approx \pi k(k=\overline{0, m})$, i.e., wherever the DR-II in $A_{m}$ is effectively valid, the DR-I holds true as well. More interesting is the value $p \approx-1$ observed for $\theta_{m} \approx \pi k-\pi / 2$ $(k=\overline{1, m})$, which shows that at frequencies characterized by prominent DR-II violation in $A_{m}$, the DR-I yet provides a reasonable estimation of $\operatorname{Im}\left[A_{m}\right]$, but with the opposite sign.

While the derived parameter $p$ gives some insight into the general relation between $\operatorname{Im}\left[A_{m}\right]$ and $\operatorname{DR}\left[\operatorname{Re}\left[A_{m}\right]\right]$, it takes on unrestrictedly large values for $\operatorname{Im}\left[A_{m}\right]=0$ (for $\left.\beta_{m}=\theta_{m}\right)$ and, hence, could not be used as a practical measure of the DR-I violation in $A_{m}$. For this purpose, it is reasonable to use the normalized DR-I violation parameter instead:

$$
\widetilde{\operatorname{Im}}^{n}[F]=\frac{\operatorname{Im}[F]-\mathrm{DR}[\operatorname{Re}[F]]}{|F|} .
$$

Substitution of Eq. (25) into Eq. (28) yields:

$$
\begin{aligned}
\widetilde{\operatorname{Im}}^{n}\left[A_{m}\right] & \approx \frac{2 \operatorname{Im}\left[A_{m}\right]-2 \operatorname{Re}\left[A_{m}\right] \beta_{m}}{\left|A_{m}\right|} \\
& =2\left(\sin \varphi_{m}-\beta_{m} \cos \varphi_{m}\right) .
\end{aligned}
$$

Again, assuming $\beta_{m}$ to be relatively small and making use of Eq. (23), we obtain

$$
\widetilde{\operatorname{Im}}^{n}\left[A_{m}\right] \approx 2 \sin \left(\varphi_{m}-\beta_{m}\right)=-2 \sin \left(\theta_{m}\right)
$$

The resulting equation states that the DR-I in $A_{m}$ is to be generally violated by no more than $\sim 200 \%$ of its amplitude, and this maximum violation be observed at each of the $n$ frequencies where $\theta_{m} \approx \pi k-\pi / 2(k=\overline{1, m})$, i.e., where $\operatorname{Re}\left[A_{m}\right] \approx 0$ and $\operatorname{DR}\left[\operatorname{Re}\left[A_{m}\right]\right] \approx-\operatorname{Im}\left[A_{m}\right]$.

Though based on a number of simplifying assumptions not supposed to be valid in the general case, the above conclusions show reasonable agreement with most synthetic and field data (presented in the second part of the paper) and hence, appear to be of rather broad applicability. However, there probably exists a more accurate formulation for the general behavior of the DR-I violation in non-causal functions, which is either yet to be derived or already known in some field of expertise we are unfamiliar with.

Received: 15 October 2019 Accepted: 11 January 2020 Published online: 03 February 2020

\section{References}

Aleksanova E, Kulikov V, Shustov N, Yakovlev A (2018) Aleksandrovka geophysical field camp: a place for probing new EM technologies. In: Paper presented at the 24th EM Induction Workshop, Helsingor, Denmark, 13-20 August 2018

Alekseev DA, Palshin NA, Varentsov IM (2009) Magnetotelluric dispersion relations in a two-dimensional model of the coastal effect. Izv Phys Solid Earth 45:167-170. https://doi.org/10.1134/S1069351309020062

Basokur AT (1999) Properties of the magnetotelluric frequency-normalised impedance over a layered medium. J Balkan Geophys Soc 2:63-74

Bechhoefer J (2011) Kramers-Kronig, Bode, and the meaning of zero. Am J Phys 79:1053-1059. https://doi.org/10.1119/1.3614039

Berdichevsky MN (1999) Marginal notes on magnetotellurics. Surv Geophys 20:341-375. https://doi.org/10.1023/A:1006645715819

Berdichevsky MN, Dmitriev VI (2002) Magnetotellurics in the context of the theory of ill-posed problems (ed and trans: Keller GV). In: Investigations in Geophysics 11. SEG, Tulsa

Berdichevsky MN, Dmitriev VI (2008) Models and methods of magnetotellurics. Springer, Berlin

Berdichevsky MN, Pokhotelov DO (1997) Dispersion relations in the magnetotelluric impedance of polarizable medium. Phiz Zemly 7:29-32 (in Russian)

Bode HW (1945) Network analysis and feedback amplifier design. D Van Nostrand Company Inc, New York

Boehl JE, Bostick FX, Smith HW (1977) An application of the Hilbert transform to the magnetotelluric method. Tech Rep Electr Geophys Res Lab, UT Austin

Chave AD (2012) Estimation of the magnetotelluric response function. In: Chave AD, Jones AG (eds) The magnetotelluric method: theory and practice. Cambridge University Press, Cambridge

Chave AD (2017) Estimation of the magnetotelluric response function: the path from robust estimation to a stable maximum likelihood estimator. Surv Geophys 38:837-867. https://doi.org/10.1007/s10712-017-9422-6

Chave AD, Thomson DJ (2004) Bounded influence magnetotelluric response function estimation. Geophys J Int 157:988-1006. https://doi. org/10.1111/j.1365-246X.2004.02203.x

Chouteau M, Tournerie B (2000) Analysis of magnetotelluric data showing phase rolling out of quadrant. In: Abstracts of the 70th SEG Annual Meeting, Calgary, Canada, 6-11 August 2000. https://doi. org/10.1190/1.1816062

Egbert GD (1990) Comments on "Concerning dispersion relations for the magnetotelluric impedance tensor" by E. Yee and K.V. Paulson. Geophys J Int 102:1-8. https://doi.org/10.1111/j.1365-246X.1990.tb00525.x

Egbert GD, Booker JR (1986) Robust estimation of geomagnetic transfer functions. Geophys J Int 87:173-194. https://doi.org/10.1111/j.1365246X.1986.tb04552.x

Egbert GD, Booker JR (1989) Multivariate analysis of geomagnetic array data: 1. The response space. J Geophys Res 94(B10):14227-14247. https ://doi.org/10.1029/JB094iB10p14227

Epishkin DV (2016) Improving magnetotelluric data-processing methods. Moscow Univ Geol Bull 71:347-354. https://doi.org/10.3103/S0145 875216050057

Ernst T, Sokolova EY, Varentsov IM, Golubev NG (2001) Comparison of two techniques for magnetotelluric data processing using synthetic data sets. Acta Geophys Polonica 49(2):213-243

Fischer G, Schnegg PA (1980) The dispersion relations of the magnetotelluric response and their incidence on the inversion problem. Geophys J Int 62:661-673. https://doi.org/10.1111/j.1365-246X.1980.tb02598.x

Fischer G, Schnegg PA (1993) The magnetotelluric dispersion relations over 2-D structures. Geophys J Int 115:1119-1123. https://doi.org/10.1111/ j.1365-246X.1993.tb01513.x

Gradstein IS, Ryzhik IM (2007) Table of integrals, series, and products. 7th edition. In: Jeffrey A, Zwillinger D, editors. Elsevier, Amsterdam 
Heise W, Pous J (2003) Anomalous phases exceeding $90^{\circ}$ in magnetotellurics: anisotropic model studies and a field example. Geophys J Int 155:308-318. https://doi.org/10.1046/j.1365-246X.2003.02050.x

Ichihara H, Mogi T (2009) A realistic 3-D resistivity model explaining anomalous large magnetotelluric phases: The L-shaped conductor model. Geophys J Int 179:14-17. https://doi.org/10.1111/j.1365-246X.2009.04310.x

Jones AG, Spratt J (2002) A simple method for deriving the uniform field MT responses in auroral zones. Earth Planets Space 54:443-450. https://doi. org/10.1186/BF03353035

Kapinos G, Brasse H (2011) Offshore magnetotellurics in the presence of bathymetry. In: Proc. of the 24th Schmucker-Weidelt Colloquium, pp 113-117

Kaufman AA, Alekseev D, Oristaglio M (eds) (2014) Principles of EM methods in surface geophysics. Methods in geochemistry and geophysics, vol 45 Elsevier, Amsterdam

Kruglyakov M, Kuvshinov A (2019) 3-D inversion of MT impedances and intersite tensors, individually and jointly: new lessons learnt. Earth Planets Space 71:4. https://doi.org/10.1186/s40623-018-0972-8

Kulikov V, Zaytsev S, Aleksanova E, Varentsov I, Lozovsky I, Shustov N, Yakovlev A (2018) Resistivity image of Baryatinskaya crustal high-conductive anomaly based on the results of areal MT-survey. In: Paper presented at the 24th EM Induction Workshop, Helsingor, Denmark, 13-20 August 2018

Kunetz G (1972) Processing and interpretation of magnetotelluric soundings. Geophysics 37:1005-1021. https://doi.org/10.1190/1.1440310

Landau LD, Lifshitz EM (1958) Statistical physics (trans Sykes JB, Kearsley MJ). Course of theoretical physics, 5th edn. Pergamon Press, Oxford

Landau LD, Lifshitz EM (1960) Electrodynamics of the continuous media (trans Sykes JB, Bell JS). Course of theoretical physics, 8th edn. Pergamon Press, Oxford

Larsen JC (1989) Transfer functions: smooth robust estimates by least-squares and remote reference methods. Geophys J Int 99:645-663. https://doi. org/10.1111/j.1365-246X.1989.tb02048.x

Lezaeta P, Haak V (2003) Beyond magnetotelluric decomposition: induction, current channeling, and magnetotelluric phases over $90^{\circ}$. J Geophys Res 108(B6):2305. https://doi.org/10.1029/2001JB000990

Marcuello A, Queralt P, Ledo J (2005) Applications of dispersion relations to the geomagnetic transfer function. Phys Earth Planetary Inter 150:85-91. https://doi.org/10.1016/j.pepi.2004.08.016

Marti A (2014) The role of electrical anisotropy in magnetotelluric responses: from modelling and dimensionality analysis to inversion and interpretation. Surv Geophys 35:179-218. https://doi.org/10.1007/s1071 2-013-9233-3

Murphy BS, Egbert GD (2018) Source biases in midlatitude magnetotelluric transfer functions due to Pc3-4 geomagnetic pulsations. Earth Planets Space 70:12. https://doi.org/10.1186/s40623-018-0781-0

Nussenzweig HM (1972) Causality and dispersion relations. Academic Press, New York

Pek J (2009) Effects of electrical anisotropy upon magnetotelluric data: modelling and experiments. In: Spichak VV(ed) Modern methods of electromagnetic data measurement, processing and interpretation. Librokom Publ, Moscow (in Russian)

Shimizu H, Yoneda A, Baba K, Utada H, Palshin NA (2011) Sq effect on the electromagnetic response functions in the period range between $10^{4}$ and $10^{5}$ s. Geophys J Int 186:193-206. https://doi.org/10.1111/j.1365246X.2011.05036.X

Simpson F, Bahr K (2005) Practical magnetotellurics. Cambridge University Press, Cambridge
Sims WE, Bostick FX, Smith HW (1971) The estimation of magnetotelluric impedance tensor elements from measured data. Geophysics 36:938942. https://doi.org/10.1190/1.1440225

Sutarno D, Vozoff K (1991) Phase-smoothed robust M-estimation of magnetotelluric impedance functions. Geophysics 56:1999-2007. https://doi. org/10.1190/1.1443012

Svetov BS (1991) Transfer functions of the electromagnetic field. Izv Acad Nauk SSSR Fiz Zemly 1:119-128 (in Russian)

Thiel S, Heinson G, Gray DR, Gregory RT (2009) Ophiolite emplacement in NE Oman: constraints from magnetotelluric sounding. Geophys J Int 176:753-766. https://doi.org/10.1111/j.1365-246X.2008.04053.x

Titchmarsh EC (1948) Theory of Fourier integrals, 2nd edn. Clarendon press, Oxford

Toll JS (1956) Causality and the dispersion relation: logical foundations. Phys Rev 104:1760-1770. https://doi.org/10.1103/PhysRev.104.1760

Utada H (2018) Plane-wave and flat Earth approximations in natural-source electromagnetic induction studies. Bull Earthq Res Inst Univ Tokyo 93:1-14

Viljanen A (2012) Description of the magnetospheric/ionospheric sources. In: Chave AD, Jones AG (eds) The magnetotelluric method: theory and practice. Cambridge University Press, Cambridge. https://doi.org/10.1017/ cbo9781139020138.005

Weckmann U, Ritter O, Haak V (2003) A magnetotelluric study of the Damara Belt in Namibia 2. MT phases over $90^{\circ}$ reveal the internal structure of the Waterberg Fault/Omaruru Lineament. Phys Earth Planetary Inters 138:91-112. https://doi.org/10.1016/S0031-9201(03)00079-7

Weidelt $P$ (1972) The inverse problem of geomagnetic induction. Zeitschrift fur Geophysik 38:257-289. https://doi.org/10.1093/gji/35.1.379

Weidelt P (2003) Dispersive conductors: the position of singularities of magnetotelluric transfer functions in the complex frequency plane. In: Hordt A, Stoll JB, editors. Proc. of the 20th Schmucker-Weidelt Colloquium, p. 280-289

Weidelt P, Kaikkonen P (1994) Local 1-D interpretation of magnetotelluric B-polarization impedances. Geophys J Int 117:733-748. https://doi. org/10.1111/j.1365-246x.1994.tb02466.x

Yee E, Paulson KV (1988) Concerning dispersion relations for the magnetotelluric impedance tensor. Geophys J 95:549-559. https://doi.org/10.1111/ j.1365-246X.1988.tb06703.x

Yee E, Paulson KV (1990) Reply to the "Comments on: Concerning dispersion relations for the magnetotelluric impedance tensor" by G.D. Egbert. Geophys J Int 102:9-13. https://doi.org/10.1111/j.1365-246X.1990.tb00526.x

Zorin NI, Alekseev DA (2018) Causality and dispersion relations in electrical prospecting. Russian Geol and Geophys 59:313-323. https://doi. org/10.1016/j.rgg.2018.03.009

\section{Publisher's Note}

Springer Nature remains neutral with regard to jurisdictional claims in published maps and institutional affiliations.

\section{Submit your manuscript to a SpringerOpen ${ }^{\circ}$ journal and benefit from:}

- Convenient online submission

- Rigorous peer review

- Open access: articles freely available online

- High visibility within the field

Retaining the copyright to your article

Submit your next manuscript at $\boldsymbol{\nabla}$ springeropen.com 